(6)

OPEN ACCESS

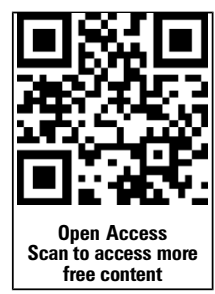

- Additional material is published online only. To view please visit the journal online (http://dx.doi.org/10.1136/ gutjnl-2014-308470).

${ }^{1}$ Stem Cells \& Cancer Group, CNIO, Madrid, Spain

${ }^{2}$ Viral Vector Unit, Spanish National Cardiovascular Research Centre (CNIC), Madrid, Spain

${ }^{3}$ Gastrointestinal Cancer

Clinical Research Unit, Spanish National Cancer Research Centre (CNIO), Madrid, Spain ${ }^{4}$ Department of Molecular Gastrointestinal Oncology, Ruhr-University Bochum, D-44801 Bochum, Germany ${ }^{5}$ Barts Cancer Institute, Centre for Stem Cells in Cancer \& Ageing, Queen Mary University of London, London, UK

\section{Correspondence to} Dr Christopher Heeschen, Centre for Stem Cells in Cancer \& Ageing, Barts Cancer Institute, Queen Mary University of London, London EC1M 6BQ, UK c.heeschen@qmul.ac.uk

Received 22 September 2014 Revised 13 February 2015 Accepted 4 March 2015

Published Online First 17 April 2015

\section{CLlinked}

- http://dx.doi.org/10.1136/ gutjnl-2015-309503

\section{CrossMark}

\section{To cite: Cioffi M,}

Trabulo SM, Sanchez-

Ripoll Y, et al. Gut

2015:64:1936-1948.

\title{
The miR-17-92 cluster counteracts quiescence and chemoresistance in a distinct subpopulation of pancreatic cancer stem cells
}

\author{
Michele Cioffi, ${ }^{1}$ Sara M Trabulo, ${ }^{1}$ Yolanda Sanchez-Ripoll, ${ }^{1}$ Irene Miranda-Lorenzo, ${ }^{1}$ \\ Enza Lonardo, ${ }^{1}$ Jorge Dorado, ${ }^{1}$ Catarina Reis Vieira, ${ }^{1,2}$ Juan Carlos Ramirez, ${ }^{2}$ \\ Manuel Hidalgo, ${ }^{3}$ Alexandra Aicher, ${ }^{1}$ Stephan Hahn, ${ }^{4}$ Bruno Sainz Jr, ${ }^{1}$ \\ Christopher Heeschen ${ }^{1,5}$
}

\section{ABSTRACT}

Objective Cancer stem cells (CSCs) represent the root of many solid cancers including pancreatic ductal adenocarcinoma, are highly chemoresistant and represent the cellular source for disease relapse. However the mechanisms involved in these processes still need to be fully elucidated. Understanding the mechanisms implicated in chemoresistance and metastasis of pancreatic cancer is critical to improving patient outcomes.

Design Micro-RNA (miRNA) expression analyses were performed to identify functionally defining epigenetic signatures in pancreatic CSC-enriched sphere-derived cells and gemcitabine-resistant pancreatic CSCs.

Results We found the miR-17-92 cluster to be downregulated in chemoresistant CSCs versus non-CSCs and demonstrate its crucial relevance for CSC biology. In particular, overexpression of miR-17-92 reduced CSC self-renewal capacity, in vivo tumourigenicity and chemoresistance by targeting multiple NODAL/ACTIVIN/ TGF- $\beta 1$ signalling cascade members as well as directly inhibiting the downstream targets p21, p57 and TBX3. Overexpression of miR-17-92 translated into increased CSC proliferation and their eventual exhaustion via downregulation of p21 and p57. Finally, the translational impact of our findings could be confirmed in preclinical models for pancreatic cancer.

Conclusions Our findings therefore identify the miR17-92 cluster as a functionally determining family of miRNAs in CSCs, and highlight the putative potential of developing modulators of this cluster to overcome drug resistance in pancreatic CSCs.

\section{INTRODUCTION}

Pancreatic ductal adenocarcinoma (PDAC) is the deadliest solid cancer and currently the fourth most frequent cause of cancer-related deaths worldwide ${ }^{1}$ and it has been predicted that by 2030 PDAC will represent the second most frequent cause of cancerrelated death. ${ }^{2}$ Despite expanding research efforts, there has been little substantial therapeutic progress towards improving clinical outcome. Current therapies, including the recently approved drug Nab-paclitaxel (Abraxane), ${ }^{3}$ only slightly improve median survival and only rarely result in long-term progression-free survival. Therefore, comprehensive

\section{Significance of this study}

What is already known on this subject?

- Pancreatic cancer is the most lethal cancer with limited therapeutic options.

- Pancreatic cancer stem cells (CSCs) are exclusively tumourigenic and highly resistant to chemotherapy.

- NODAL/ACTIVIN/TGF- $\beta 1$ pathway is composed of a core group of regulatory genes that govern the stemness and metastatic activity of pancreatic CSCs.

What are the new findings?

- miR-17-92 cluster is downregulated in (chemoresistant) CSCs.

- NODAL/ACTIVIN/TGF- $\beta 1$ signalling in CSCs promotes their chemoresistance, which is inhibited by the miR-17-92 cluster.

- Overexpression of miR-17-92 results in abrogation of CSC phenotypes and eventual loss of in vivo tumourigenicity.

How might it impact on clinical practice in the foreseeable future?

- The discovery of the crucial role of the miR-1792/NODAL-ACTIVIN-TGF- $\beta 1 / p 21$ Tbx3 axis in CSCs represents an important advancement in our understanding of CSC biology.

- Targeting pancreatic CSCs using miR-17-92 could be a highly specific therapeutic approach acting upstream and downstream of the NODAL/ACTIVIN/TGF- $\beta 1$ signalling cascade.

elucidation of the mechanisms governing resistance to treatment and rapid relapse in PDAC is urgently needed.

In this context, cancer stem cells (CSCs) have been identified as key players in disease progression and resistance to conventional chemotherapies for many carcinomas ${ }^{4}$ including PDAC. ${ }^{5}{ }^{6}$ Due to their hierarchical organisation, CSCs and non-CSCs share the same mutational/genetic background; however, CSCs are exclusively tumourigenic and chemoresistant. Thus, epigenetic mechanisms likely 
account for the strong phenotypical differences between these two cell types. Indeed, several microRNAs (miRNAs) have already been implicated in the regulation of normal stem cells as well as CSCs, ${ }^{7-10}$ but miRs implicated in CSC chemoresistance have remained mostly elusive to date.

\section{MATERIALS AND METHODS}

\section{Additional details are provided in online supplementary} materials and methods

Primary human pancreatic cancer cells

Human pancreatic tumours were obtained with written informed consent from all patients and expanded in nude mice patientderived xenograft (PDX). ${ }^{11-13}$ For in vitro studies, tissue fragments were minced, enzymatically digested with collagenase (Stem Cell Technologies, Vancouver, British Columbia, Canada) for $90 \mathrm{~min}$ at $37^{\circ} \mathrm{C}^{14}$ and after centrifugation for $5 \mathrm{~min}$ at $1200 \mathrm{rpm}$ the pellets were resuspended and cultured in RPMI medium (Roswell Park Memorial Institute), 10\% fetal bovine serum (FBS) and 50 units/mL penicillin/streptomycin.

\section{In vivo tumourigenicity and metastasis assays}

For tumourigenicity assays, serial dilutions of single cells resuspended in Matrigel (BD Bioscience) were subcutaneously injected into female NU-Foxn $1^{n u}$ nude mice (Harlan, Laboratories, UK) and tracked for 3 months. For metastasis assays, $5 \times 10^{4}$ FACSorted mCHERRY+miR-control and miR-17-92 cells were resuspended in 1X PBS (phosphate buffered saline) and intrasplenically injected into NOD scid IL2 receptor $\gamma$ chain knockout (NSG) mice as previously described. ${ }^{15}$ For serial transplantation experiments, excised tumours were digested and sorted for green fluorescent protein (GFP) and implanted again using equal number of cells. Mice were housed according to institutional guidelines and all experiments were approved by the Animal Experimental Ethics Committee of the Instituto de Salud Carlos III (Madrid, Spain) and performed in accordance with the guidelines for Ethical Conduct in the Care and Use of Animals as stated in The International Guiding Principles for Biomedical Research involving Animals, developed by the Council for International Organizations of Medical Sciences (CIOMS).

\section{Drugs, recombinant proteins and inhibitors}

Gemcitabine (Gemzar, Lilly SA, Alcobendas, Spain) was resuspended to a working concentration of $1 \mu \mathrm{g} / \mathrm{mL}$ in PBS. Recombinant NODAL, ACTIVIN A and TGF- $\beta 1$ were purchased from R\&D Systems and resuspended according to the manufacturer's recommendations.

\section{In vivo treatment of established pancreatic cancers}

Two $\mathrm{mm}^{3}$ pieces of low-passage xenograft tissue derived from patients with histologically confirmed PDAC ${ }^{11-13}$ were implanted subcutaneously into NU-Foxn $1^{n u}$ nude mice (Harlan), and mice were randomised to the respective treatment groups. Size and weight of the pancreatic tumours were monitored. Gemcitabine was administered twice a week $(125 \mathrm{mg} / \mathrm{kg} / \mathrm{mouse}$ intraperitoneally). Doxycycline was administered in drinking water twice a week at a concentration of $2 \mathrm{mg} / \mathrm{mL}$.

More Materials and Methods can be found as online supplementary information.

\section{RESULTS}

\section{Enrichment strategy for primary chemoresistant CSCs}

To identify miRNA profiles that are most representative of human pancreatic CSCs we used two mutually complementary approaches: First, we used anchorage-independent cultures of primary PDAC cells (ie, spheres) to globally enrich for CSCs (see figure $1 \mathrm{~A}, \mathrm{~B}$ and online supplementary figure S1A). ${ }^{5}{ }^{16}$ Second, CSC-enriched sphere cultures were treated with the standard chemotherapeutic gemcitabine to further enrich for the CSC population via depletion of their more differentiated progenies (see figure 1C, D and online supplementary figure S1B). Consistently, mRNA expression of the NODAL/ACTIVIN/ TGF- $\beta 1$ pathway members ALK4, TGFBRII, SMAD2, SMAD4 and TBX3, which we have previously shown to be crucial for CSC function, ${ }^{16}$ was increased in chemoresistant CSCs (see online supplementary figure S1C). We also noted differential expression of cellular transporters implicated in drug resistance, ${ }^{17}{ }^{18}$ such as upregulation of the ABC-transporters ABCC1 and ABCG2 and downregulation of the gemcitabine-specific transporters human concentrative nucleoside transporter and human equilibrative nucleoside transporter (see online supplementary figure S1D), both of which are mandatory for gemcitabine uptake. ${ }^{19}$ These data were then validated in vivo using the original patient-derived xenografts (PDXs). PDXs were treated with vehicle or gemcitabine (figure 1E), dissociated into single cell suspension, and depleted for contaminating mouse stroma cells (see online supplementary figure S1E). As predicted by our in vitro data, CSCs were enriched following gemcitabine treatment (figure $1 \mathrm{~F}-\mathrm{H}$ ) and mRNA expression for members of the NODAL/ACTIVIN/TGF- $\beta 1$ pathway was also enhanced (figure 1I).

\section{Identification of quiescent and chemoresistant CSCs}

In the CSC-enriched fraction isolated from several PDX models we found that the cell cycle regulators p21 and p57 were consistently upregulated and Cyclin D1 downregulated, as compared with the more differentiated non-CSC progenies, at RNA and protein levels (figure 2A,B). Functionally, CSCs showed a significant reduction of cells in $\mathrm{S}$ phase and G2-M phase, which was accompanied by a notable enrichment for cells in G0 and G1 phases (figure 2C) and enhanced chemoresistance to gemcitabine (figure 2D). Gemcitabine-treated CSCs showed significant enrichment for cells residing in G0 and G1, which was accompanied by a decrease of cells in $S$ phase (figure 2E) and higher mRNA levels of p21 (cyclin-dependent kinase inhibitor 1A) and p57 (cyclin-dependent kinase inhibitor 1C) as well as downregulation of cyclin-D1 (figure 2F). To further validate the presence of a slow cycling and chemoresistant CSC subpopulation, we stained cells with PKH26 2021 and found that only 2-3\% of the originally PKH26-labelled cells had retained strong dye labelling after 4 weeks, indicating that those rare cells had yet to divide (figure 2G and data not shown). Importantly, label-retaining cells were not senescent, but rather showed enhanced sphere formation capacity as compared with label-negative cells (see online supplementary figure S2B) and also expressed higher levels of the CSC surface markers (see online supplementary figure S2A). As these cells were also highly resistant to gemcitabine (figure $2 \mathrm{H}$ ) our data suggest that within the heterogeneous population of CSCs a subpopulation of quiescent and highly chemoresistant CSCs exists and we thus performed further in-depth investigations to mechanistically dissect these cells.

\section{miR-17-92 is suppressed in quiescent and chemoresistant CSCs}

In order to identify epigenetic regulators of more quiescent and thus chemoresistant CSCs, we compared miRNA expression profiles of (1) CSC-enriched sphere-derived cells with adherent cells and (2) Gemcitabine-treated PDXs with vehicle-treated control tumours. Importantly, our experiments were designed to identify miRNAs that bear relevance for CSCs across broad 

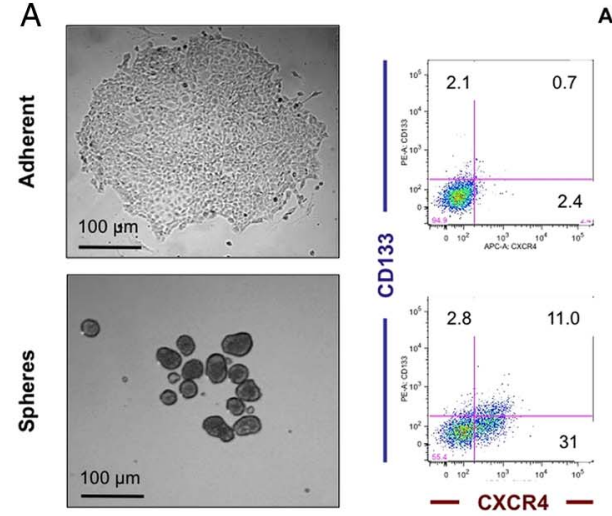

商

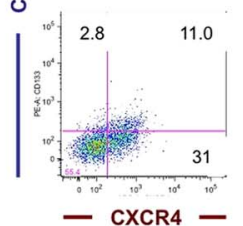

A6L
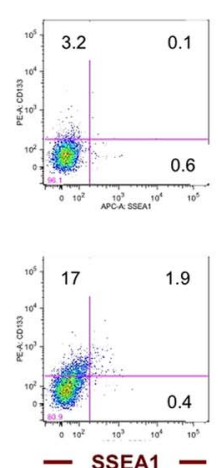

B

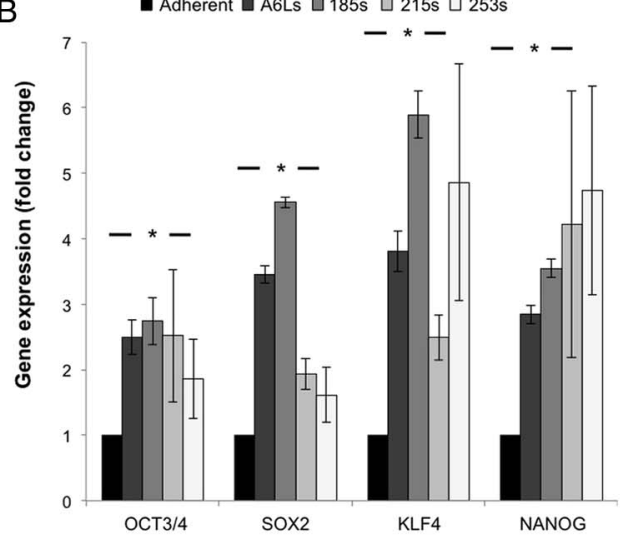

C

일

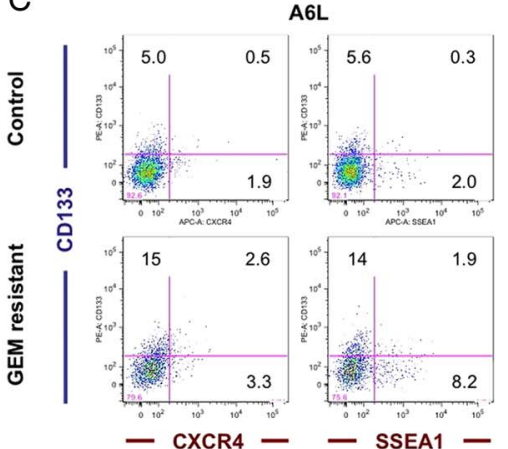

E

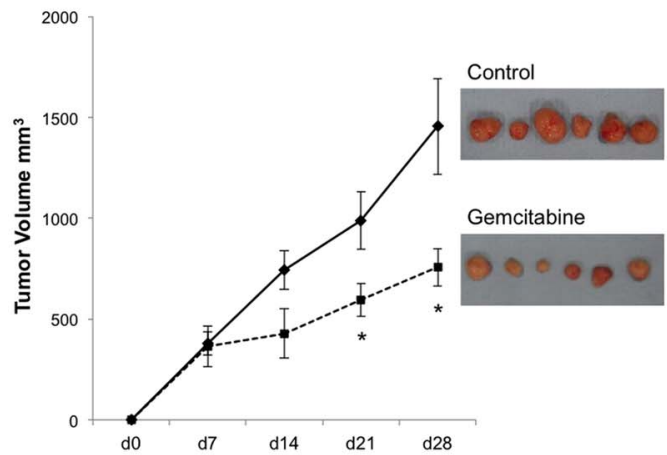

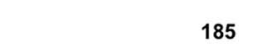
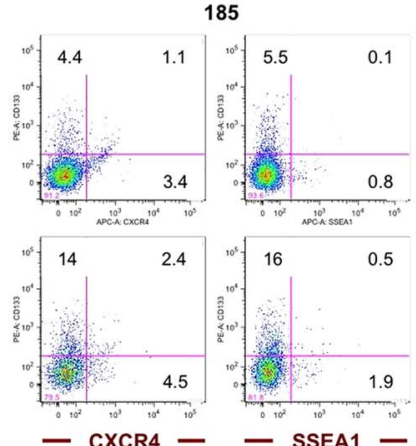

$\mathrm{F}$

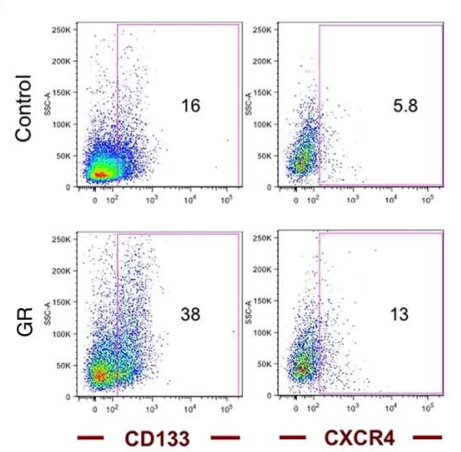

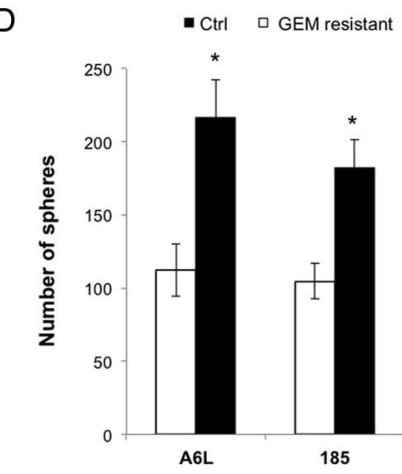

G

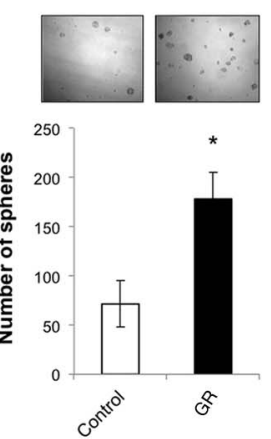

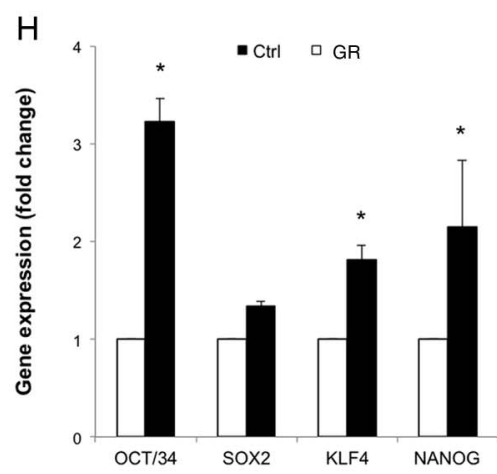

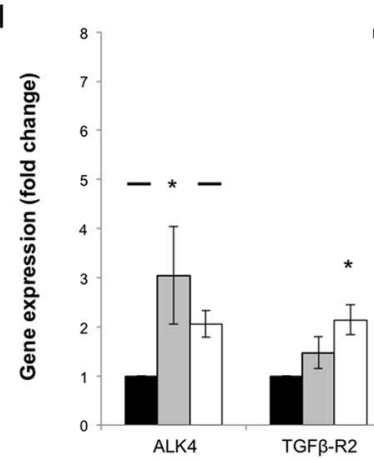

- Ctrl a A6L GR $\quad 185 \mathrm{GR}$
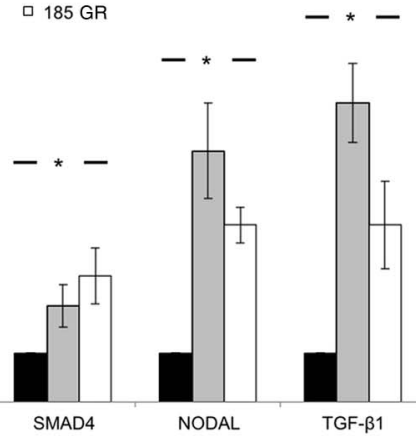

Figure 1 Enrichment strategies for cancer stem cells. (A) Representative pictures of primary pancreatic ductal adenocarcinoma (PDAC) cells cultured as adherent monolayers or as spheres (s) (left panel). Flow cytometry analysis of CD133+CXCR4+ and CD133+SSEA1+ expression (right panel). (B) RTqPCR analysis of pluripotency-associated genes Oct4, Sox2, Klf4 and Nanog. Data are normalised for B-Actin expression ( $n=3$; $\left.{ }^{*} p<0.05\right)$. (C) Presence of CD133+CXCR4+ and CD133+SSEA1+ cells as assessed by flow cytometry in control and gemcitabine resistant (GR) cells established from primary PDAC A6L and 185 cultures (D) Sphere formation capacity $\left(n=3 ;{ }^{*} p<0.05\right)$. (E) Patient-derived xenografts were treated with vehicle or gemcitabine $(125 \mathrm{mg} / \mathrm{kg} /$ mouse biweekly; treatment from day 7 to day 28$)$. Tumour diameters were measured using callipers, and volumes in $\mathrm{mm}^{3}$ were calculated $\left(\mathrm{n}=6 ;{ }^{*} \mathrm{p}<0.05\right)$. ( $\left.\mathrm{F}-\mathrm{I}\right)$ Epithelial cells isolated from explanted/digested control-treated and gemcitabine resistant tumours were analysed. (F) Flow cytometry analysis for CD133 and CXCR4 cell surface expression. (G) Sphere formation capacity. Each bar represents mean sphere number $\pm \mathrm{SD}\left(\mathrm{n}=3 ;{ }^{*} \mathrm{p}<0.05\right)$. Original magnification $\times 100$. (H) RTqPCR analysis of pluripotency-associated genes and (I) genes involved in NODAL/ACTIVIN signalling (ALK4, NODAL, SMAD2, SMAD4, TGF- $\beta$-RII and TGF- $\beta 1)\left(n=3 ;{ }^{*} p<0.05\right)$. 


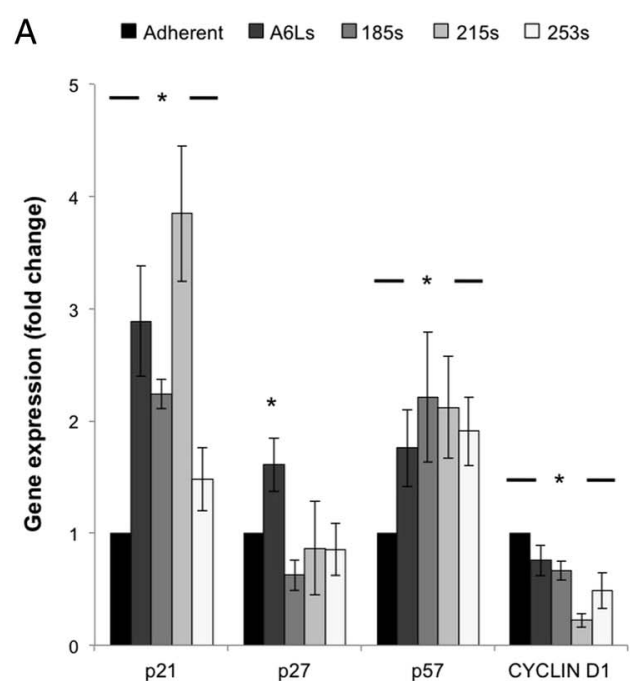

D
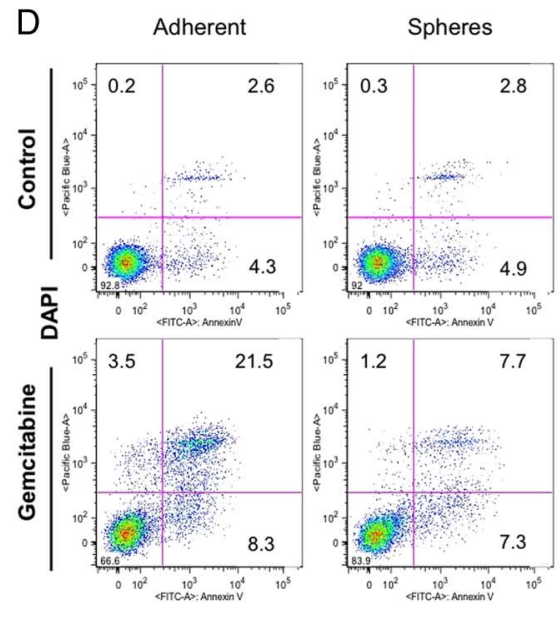

Annexin V
B

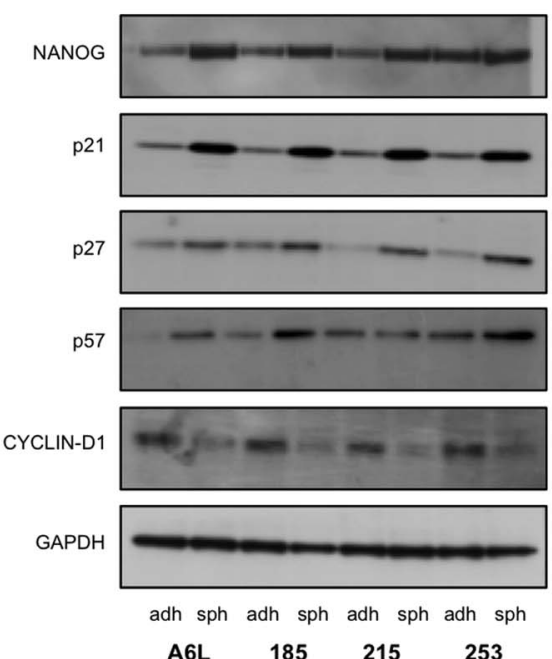

E

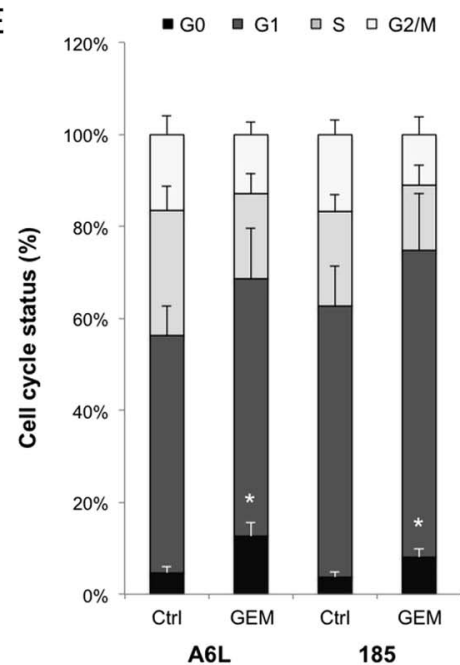

$\mathrm{F}$
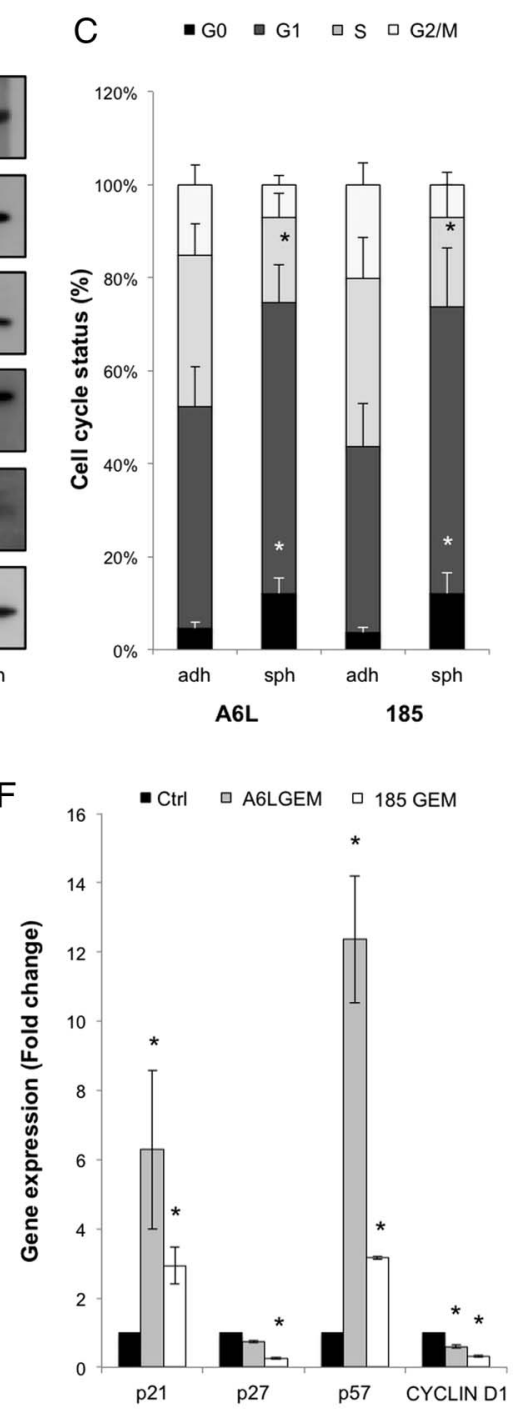

$\mathrm{H}$

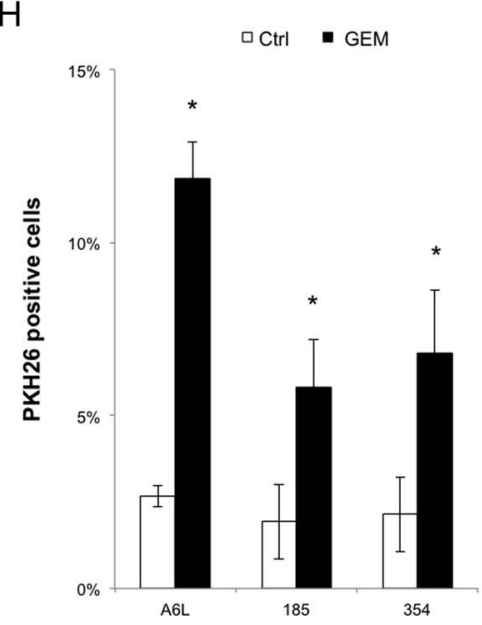

Figure 2 Identification of quiescent and chemoresistant cancer stem cells. (A) RTqPCR analysis of pluripotency-associated genes and genes involved in cell cycle regulation p21, p27, p57, Cyclin-D1. Data are normalised for B-Actin expression ( $n=3$; * $p<0.05)$. (B) Western blot analysis of NANOG and cell cycle proteins p21, p27, p57 and CYCLIN-D1. Adherent (adh); sphere-derived cells (sph). (C) Cell cycle analysis using Ki67 and DAPI staining $\left(n=3 ;{ }^{*} p<0.05\right)$. (D) Flow cytometry analysis for apoptotic cells following $48 \mathrm{~h}$ of treatment with gemcitabine $(100 \mathrm{ng} / \mathrm{mL})$ as determined with AnnexinV/DAPI staining. (E) Cell cycle analysis using Ki67 and DAPI of gemcitabine-resistant cells $\left(n=3 ;{ }^{*} p<0.05\right)$. (F) RTqPCR analysis of cell cycle genes p21, p27, p57 and Cyclin D1 ( $n=3$; $\left.{ }^{*} \mathrm{p}<0.05\right)$. (G) Representative images of PKH26 labelled cells after 1 day, 14 days and 28 days in culture (upper panel) and flow cytometry analysis (lower panel). (H) Percentage of PKH26+ cells before and after gemcitabine treatment in A6L, 185 and 354 pancreatic ductal adenocarcinoma (PDAC) tumours $\left(n=3 ;{ }^{*} p<0.05\right)$. 
populations of patients with pancreatic cancer, and as such we used a panel of primary pancreatic tumours. Of interest, we discovered a small number of miRNAs that was commonly and differentially expressed in gemcitabine-resistant CSCs (figure 3A). Surprisingly, most members of the miR-17-92 cluster, which are regularly found to be upregulated in bulk cancer tissue including pancreatic cancer, ${ }^{22}$ were also among the set of miRNAs downregulated in chemoresistant CSCs. To further collaborate these findings, we next analysed the miRNA signature of gemcitabine-resistant cells (versus vehicle-treated cells) with the miRNA signature of CSC-enriched spheres (versus adherent cells) and again found consistent and significant downregulation of members of the miR-17-92 cluster together with miR-513a-5p and miR-513b (figure 3B). Importantly, we could confirm the downregulation of the miR-17-92 family members by RTqPCR analysis using an independent set of primary cultures (see online supplementary figure S3A) as well as PKH26-positive (ie, quiescent) cells (see online supplementary figure S3B). Thus, these data provide evidence that a distinct subpopulation of slow-cycling/quiescent pancreatic CSCs exists within the PDAC tumour bulk that is characterised by low expression of the miR-17-92 cluster.

\section{Loss-of-function experiments}

We knocked down miR-17-92 in more differentiated cancer cells, which regularly overexpress miR-17-92, using antisense inhibitor of miR-17-92 (termed antagomir-17-92) in order to investigate putative alterations in stemness features and chemoresistance. Indeed, treatment with antagomir-17-92 resulted in increased expression of the CSC surface marker CD133 (figure 3C), pluripotency-associated genes and $\mathrm{ABC}$ transporters (figure 3D) as well as functionally translated into enhanced self-renewal as determined by sphere formation (figure 3E). Of note, transfected cells showed an increase in cells residing in G0 and G1 phases accompanied by a reduction of cells in $\mathrm{S}$ phase (figure $3 \mathrm{~F}$ ), a marked increase in p21 expression (see online supplementary figure $\mathrm{S} 3 \mathrm{C}$ ), and most importantly increased chemoresistance to gemcitabine (figure 3G). Of note, these cells also showed enhanced in vivo tumourigenicity as evidenced by significantly higher tumour take rates (see online supplementary figure S3D) and faster growth rates (data not shown). Taken together, inhibition of the miR-17-92 cluster in differentiated pancreatic cancer cells gave rise to cells reminiscent of bona fide CSCs with enhanced chemoresistance, supporting the hypothesis that the miR-17-92 cluster negatively controls CSC features.

\section{Gain-of-function experiments}

Adversely, overexpression of miR-17-92 in CSCs should counteract CSC stemness properties. To test this hypothesis we used a lentiviral construct expressing GFP and the common precursor of the miR-17-92 cluster (miR-17-92) or a scrambled control (miR-Ctrl). Compared with miR-Ctrl, miR-17-92 cluster overexpression led to efficient upregulation of miR-17-92 family members in CSCs (see online supplementary figure S4A) and subsequent downregulation of CSC surface markers (figure 4A), pluripotency-associated genes (see online supplementary figure S4B) and sphere formation capacity (figure 4B). Intriguingly, miR-17-92 reduced the fraction of cells residing in G0 phase and G1 phase while cells in S phase increased suggesting a less quiescent phenotype (figure 4C). Indeed, we did not observe any alterations in the level of senescence (see online supplementary figure S4C), but rather reduced quiescence, which was functionally validated by PKH26 labelling (see online supplementary figure S4D) and translated into a marked increase in chemosensitivity to gemcitabine (see online supplementary figure 4D) as well as Abraxane and 5-FU (see online supplementary figure S4E). These data demonstrated that miR-17-92 abrogated the more quiescent CSC phenotype and subsequently restored chemosensitivity. Despite the higher proliferation rate of miR-17-92 cells, long-term in vivo tumourigenicity was reduced (figure 4E). During three serial in vivo passages, the highly proliferative miR-17-92 cells gradually lost their potential to expand and eventually exhausted as observed for more differentiated cancer cells, but not for miR-Ctrl CSCs (figure 4F). Indeed, ex vivo self-renewal capacity of miR-17-92-overexpressing CSCs harvested after each in vivo passage significantly decreased as observed in sphere formation assays (see online supplementary figure S4F). Moreover, cell cycle analysis validated the initial increase in proliferation (see online supplementary figure S4G), which eventually culminated in CSC exhaustion.

Metastatic activity represents an integrative feature of a subset of CSCs. ${ }^{5}$ While miR-Ctrl CSCs responded robustly to key chemoattractant factors, TGF- $\beta 1$ and NODAL, miR-17-92 CSCs showed markedly reduced responsiveness in terms of migration (see online supplementary figure $\mathrm{S} 4 \mathrm{H}$ ) and invasion (figure 4G). As predicted by the above experiments, pharmacological inhibition of Alk4 and TGFBR2 reduced the migratory capacity of miR-Ctrl CSCs, while the additional receptor inhibition in miR-17-92 CSCs essentially ablated their entire migratory capacity. Importantly, we validated these findings in vivo by intrasplenic injection of miR-Ctrl-mCherry or miR-17-92mCherry CSCs to assess liver dissemination and subsequent development of metastasis. Ten weeks postinjection, we found significantly reduced cell dissemination in mice injected with miR-17-92 CSCs, as determined by in situ hybridisation using a human specific Alu II repeat probe (ALU) probe, immunohistochemical analysis for human cytokeratin-19 in extracted formalin-fixed paraffin embedded (FFPE) livers, and RTqPCR analysis of mCherry and hGAPDH mRNA expression in whole liver homogenates (figure 4H).

\section{miR-17-92 targets NODAL/ACTIVIN/TGF- $\beta 1$ signalling}

To begin to understand how the miR-17-92 cluster alters the CSC transcriptome, we used several computational methods to search for potential targets of miR-17-92. TargetScan (http:// www.targetscan.org) identified conserved binding sites for several members of the miR-17-92 cluster in the $3^{\prime}$-UTR (untranslated regioin) of the Activin-like 4 (ALK4 or ACVR1B), TGF- $\beta$ Receptor-2 (TGFBR2), SMAD2 and SMAD4 genes, a core group of regulatory genes known to govern the stemness and/or metastasis of pancreatic CSCs, ${ }^{16}$ p21 and p57, main regulators of quiescence $\mathrm{e}^{23-25}$ and TBX3, which has been implicated in the regulation of self-renewal of embryonic stem cells ${ }^{26}$ and breast $\mathrm{CSCs}^{27}$ (see figure 5A and online supplementary figure $\mathrm{S} 5 \mathrm{~A})$. To validate these genes as direct functional targets of miR-17-92, we analysed a panel of primary pancreatic cancer cells expressing miR-17-92 or miR-Ctrl under the control of doxycycline (see online supplementary figure S5B). Induction of miR-17-92 resulted in reduced ALK4 cell surface expression (figure 5B) as well as diminished expression of p21, p57, pSMAD2 and Tbx3 at the mRNA (figure 5C) and protein levels (figure 5D). We also validate the regulation of the predicted targets by single members of the miR-17-92 cluster by qPCR analysis following treatment with the indicated antagomirs, and while all miR-17-92 members were capable of inhibiting the target genes analysed, miR-92a showed a particular preference for only one of the identified targets (p57) (see online supplementary figure S5C). 

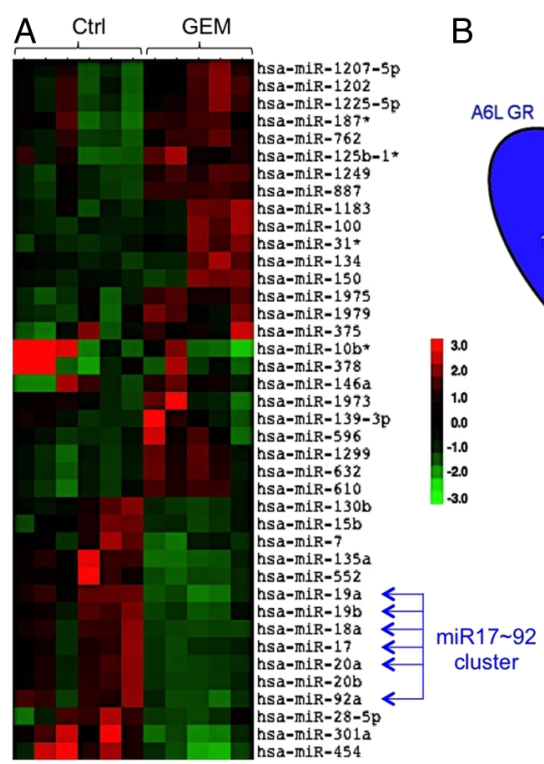

D
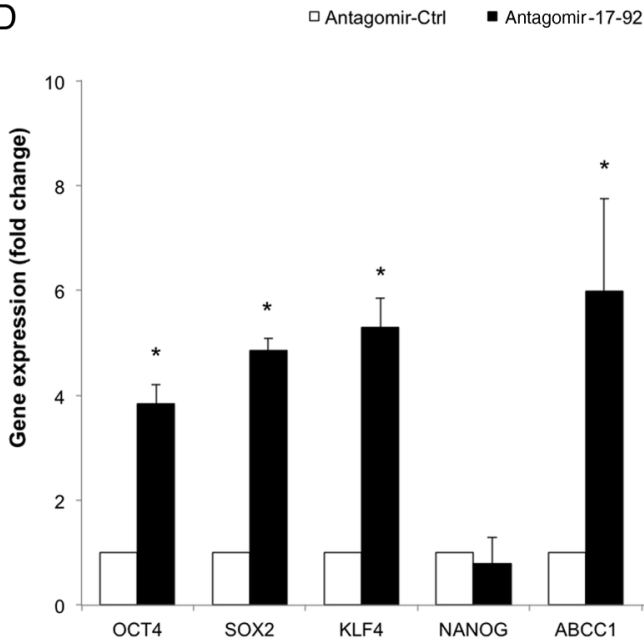

$\mathrm{F}$
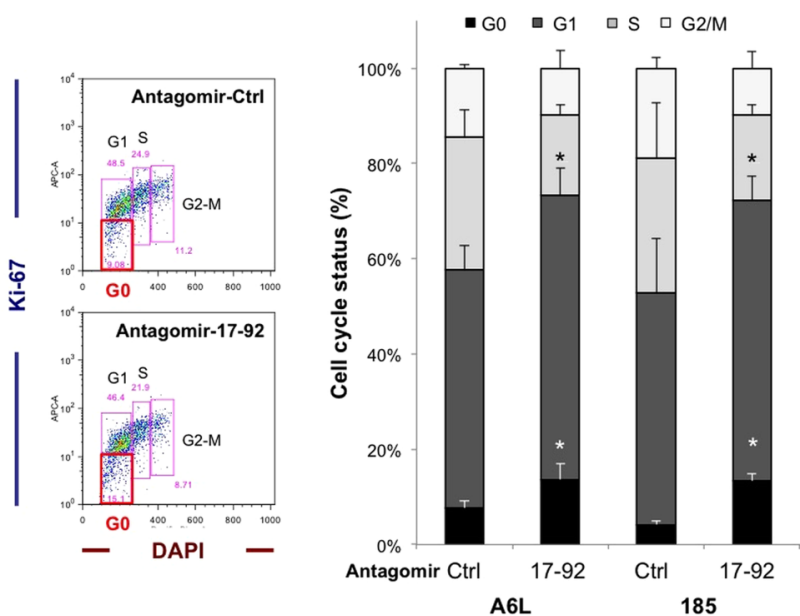

C

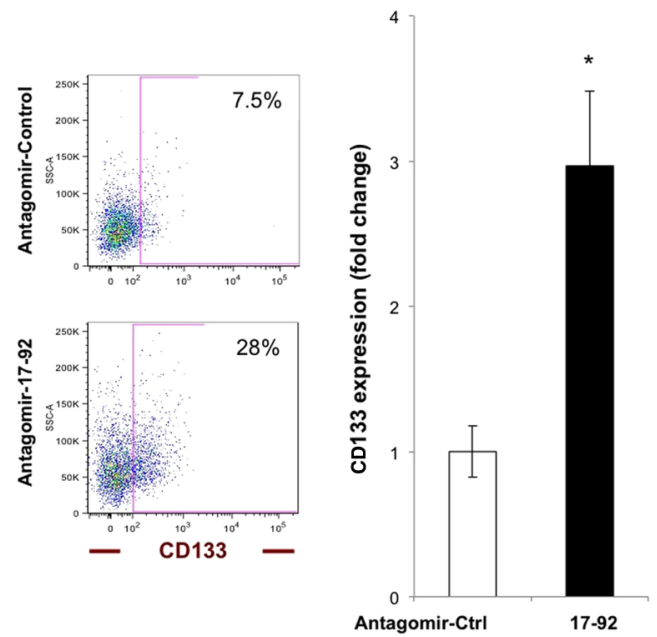

$E$

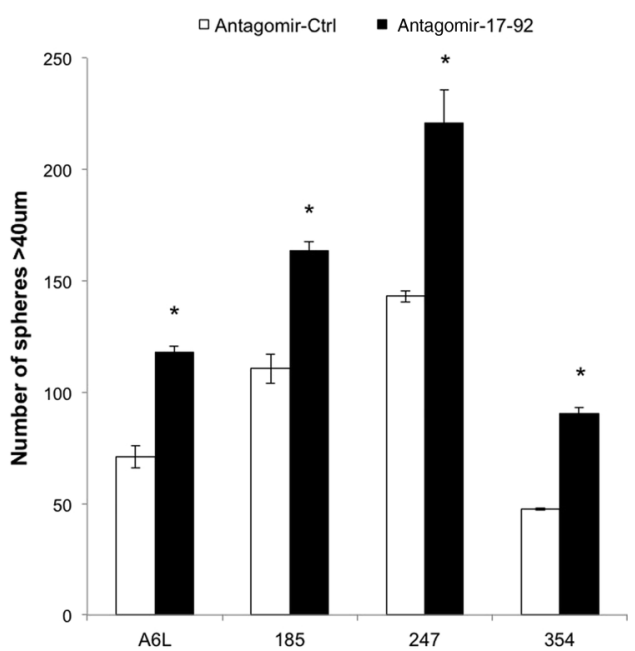

G

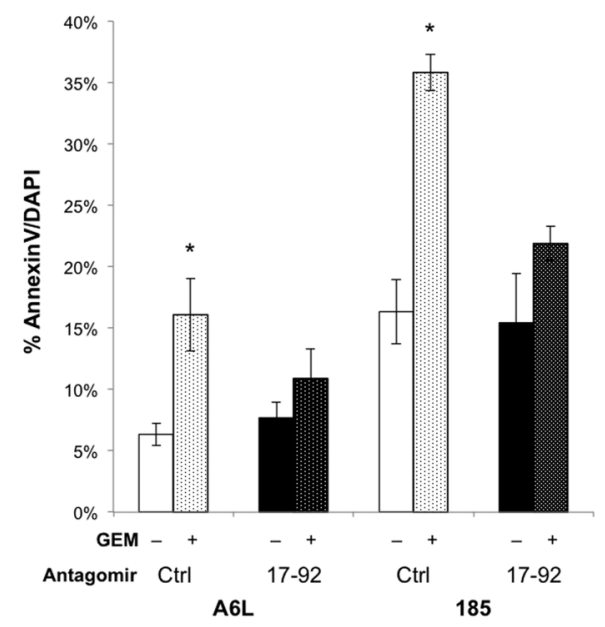

Figure 3 Inhibition of miR-17-92 promotes tumourigenicity and chemoresistance. (A) Microarray analysis represented as heat-maps, of differentially regulated miRNAs in control (Ctrl)-treated or gemcitabine (GEM)-treated pancreatic ductal adenocarcinoma (PDAC) cells (red and green boxes indicate upregulation and downregulation, respectively). (B) Venn diagram showing overlap between miRNAs downregulated in gemcitabine-resistant (GR) and sphere (SPH) cultures. (C-G) Primary PDAC adherent cultures were treated with control scrambled (SCR) antagomirs or antagomirs against the miR-17-92 cluster. (C) Flow cytometry analysis and quantification of CD133 cell surface expression ( $n=3$; * $p<0.05)$. (D) RTqPCR analysis of pluripotency-associated genes. Data are normalised for B-Actin expression $\left(n=3 ;{ }^{*} p<0.05\right)$. (E) Sphere numbers $/ \mathrm{mL}$ for four primary PDAC cultures $\left(n=3 ;{ }^{*} p<0.05\right)$. (F) Cell cycle analysis using Ki67 and DAPI $\left(n=3 ;{ }^{*} p<0.05\right)$. (G) Chemoresistance to gemcitabine determined with AnnexinV/DAPI staining after $48 \mathrm{~h}$ treatment $\left(n=3 ;{ }^{*} \mathrm{p}<0.05\right)$. 


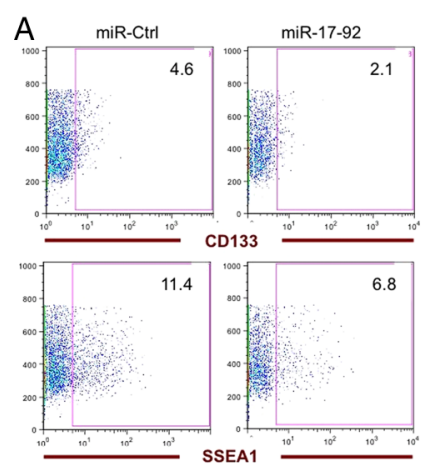

C

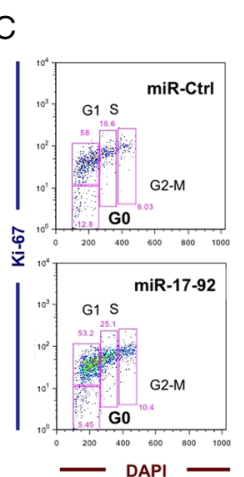

$120 \%$

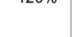

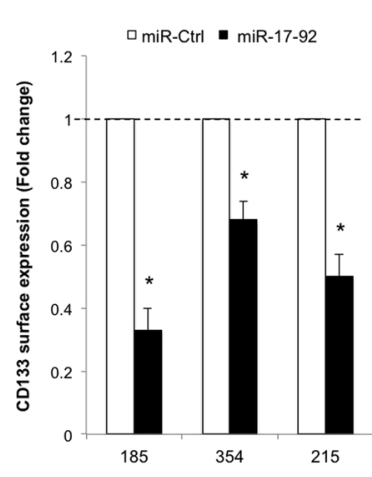

- $\mathrm{G} 0 \quad \square \mathrm{G} 1 \quad \square \mathrm{S} \quad \square \mathrm{G} 2 / \mathrm{M}$
B

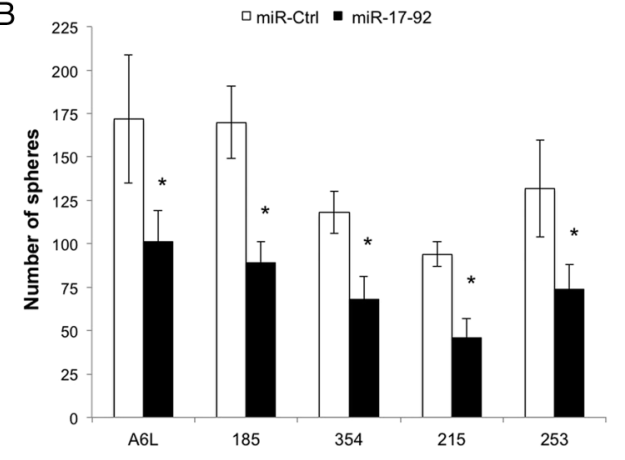

$\mathrm{D}$
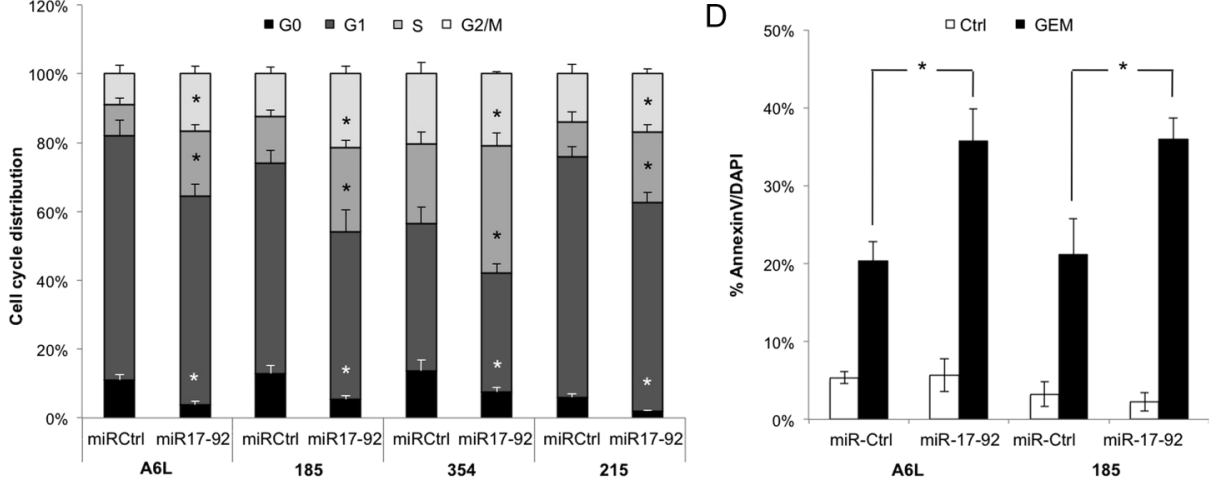

$\mathrm{E}$

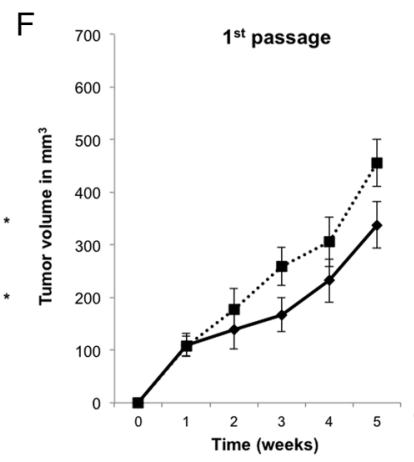

${ }^{*} p=0.001$

$2^{\text {nd }}$ passage

A6L

185

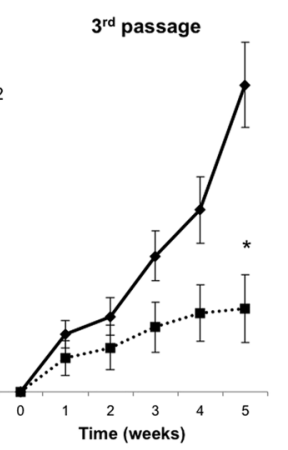

G

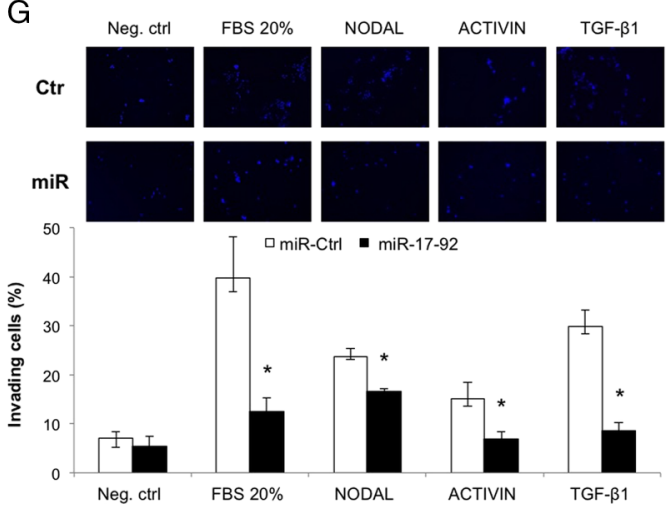

$\mathrm{H}_{215}$

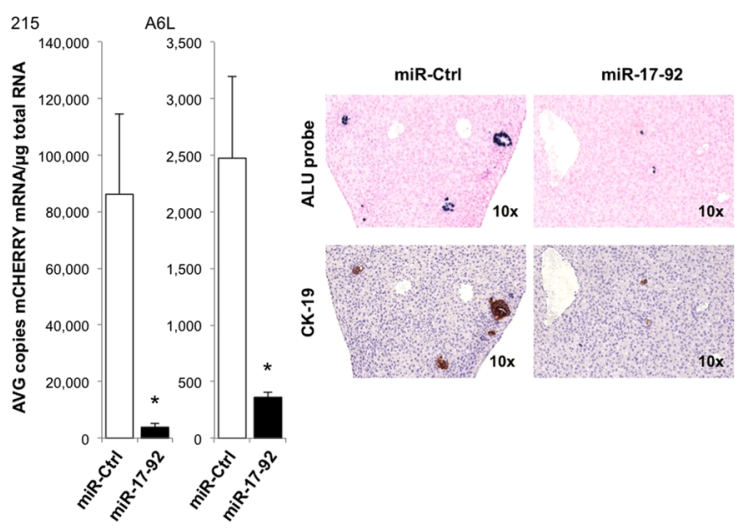

Figure 4 Overexpression of miR-17-92 reverses quiescence and chemoresistance. (A-H) Cancer stem cells (CSCs) were infected with a control lentivirus (miR-Ctrl) or a lentivirus overexpressing the pre-miR-17-92 cluster (miR-17-92) followed by comparative analyses. (A) Fold change in surface expression of CD133 and SSEA1. Dashed line represents levels obtained in miR-Ctrl samples $\left(n=3 ;{ }^{*} p<0.05\right)$. (B) Sphere formation capacity $\left(n=3 ;{ }^{*} p<0.05\right)$. (C) Cell cycle analysis using Ki67 and DAPI $\left(n=3 ;{ }^{*} p<0.05\right)$. (D) Chemoresistance by AnnexinV/DAPI staining after treatment with gemcitabine $\left(n=3 ;{ }^{*} p<0.05\right)$. (E) In vivo tumourigenicity results. CSC frequencies (Freq) determined using the extreme limiting dilution analysis algorithm (http://bioinf.wehi.edu.au/software/elda/index.html) $\left({ }^{*} \mathrm{p}<0.05\right)$. (F) In vivo serial transplantation results. Tumour volumes $\left(\mathrm{mm}^{3}\right)$ were determined at indicated times. At 5 weeks postimplantation, tumours were explanted and serially transplanted into naïve nude mice $(n=4$; ${ }^{*} \mathrm{p}<0.05$ ). (G) Representative images of invaded cells (upper panel). Percentage of cells that transmigrated through Matrigel following stimulation with NODAL, ACTIVIN or TGF-B1 (lower panel; $n=3$; ${ }^{*} p<0.05$ ). (H) RTqPCR quantification of mCHERRY mRNA copies/ $\mu \mathrm{g}$ of total RNA in fresh liver homogenates from NSG mice 10 weeks after intrasplenic injection of $5 \times 10^{4}$ miR-Ctrl or miR-17-92 mCherry-labelled cells (left panel; $\left.n=4 ;{ }^{*} p<0.05\right)$ ) and in situ hybridisation for ALU probe and immunohistochemistry for CK-19 in explanted FFPE livers (right panel). 
A

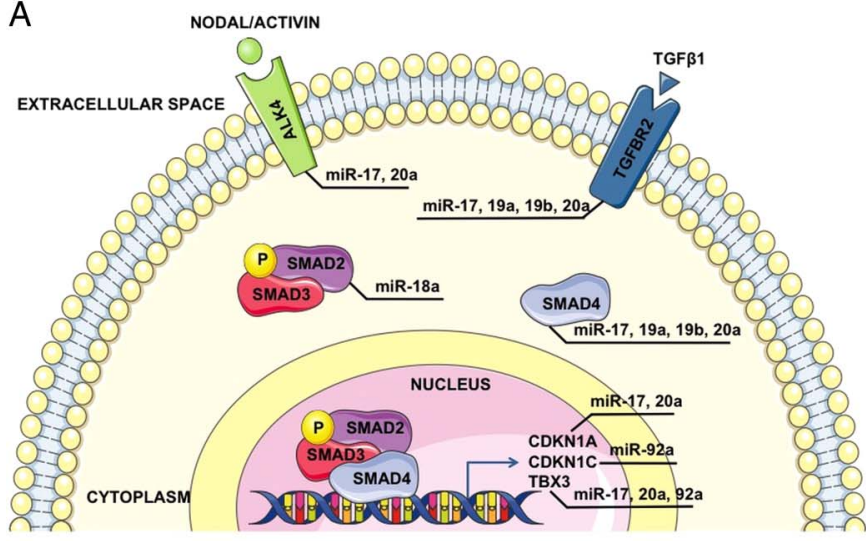

B
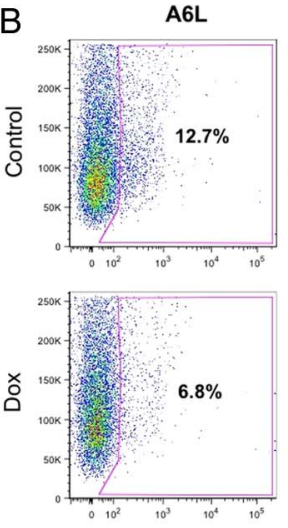

185
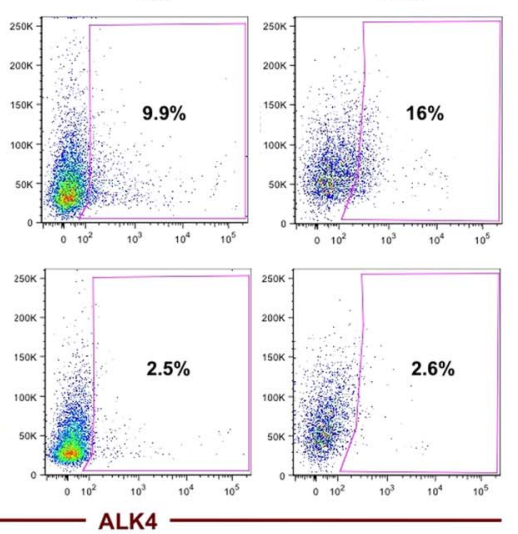

* Servier Medical Art

C

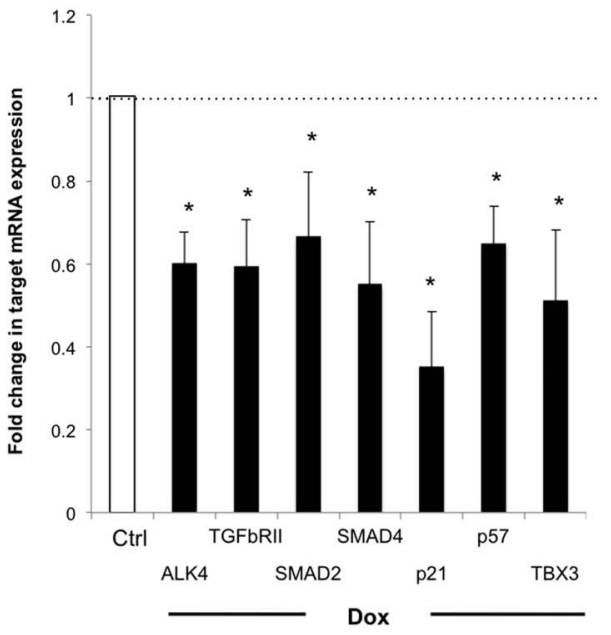

$\mathrm{F}$

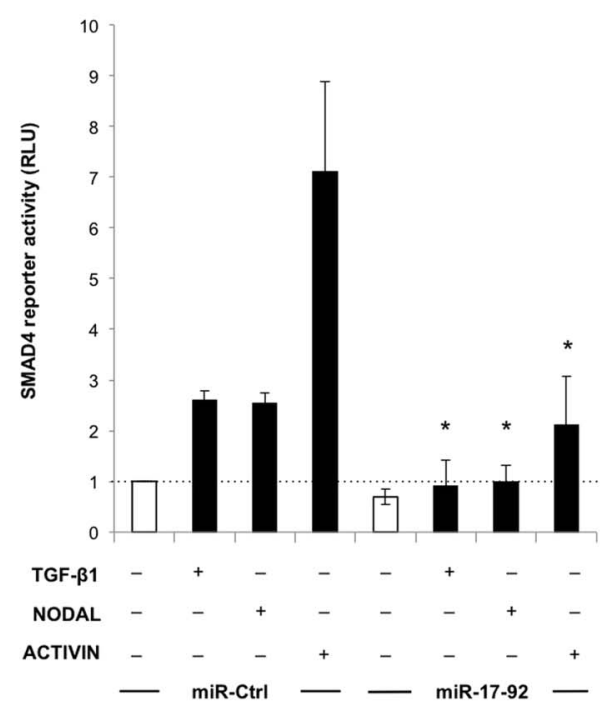

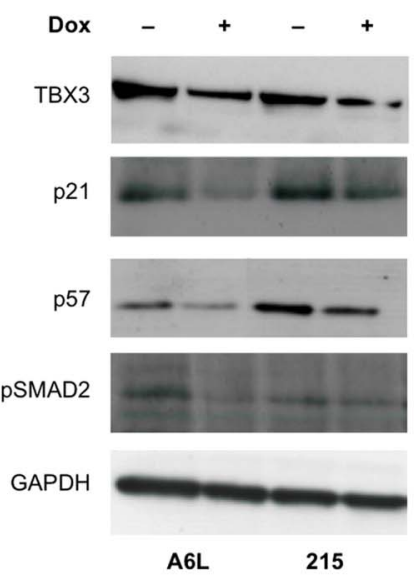

G

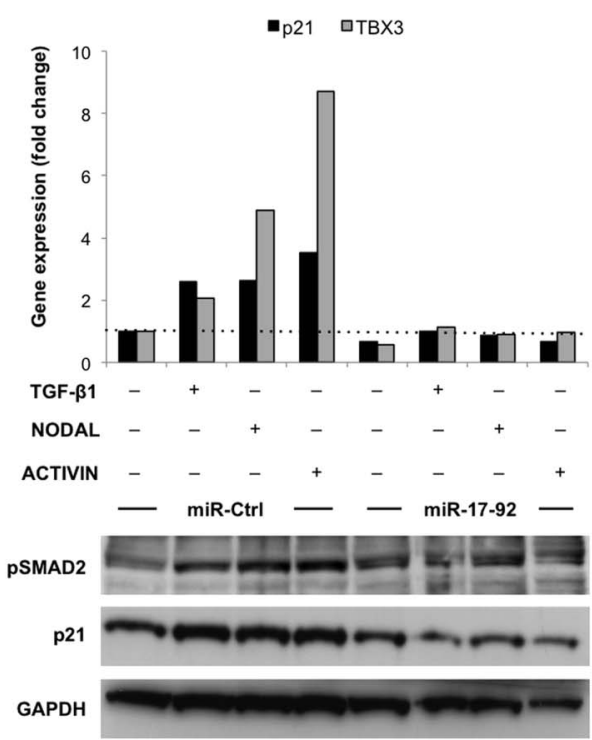

Figure 5 miR-17-92 targets NODAL/ACTIVIN/TGF- $\beta$ signalling. (A) Graphic representation of predicted targets of the miR-17-92 cluster. (B) Flow cytometry analysis for ALK4 cell surface expression before and $96 \mathrm{~h}$ after treatment with doxycycline $2 \mu \mathrm{g} / \mathrm{ml}$. (C) RTgPCR analysis of miR-17-92 target genes after $72 \mathrm{~h}$ of treatment with doxycycline. Data are normalised for B-Actin expression $\left(n=3 ;{ }^{*} p<0.05\right)$. (D) Western blot analysis of cell cycle proteins p21 and p57, pSMAD2 and TBX3. (E) The complete 3' UTR of ALK4 and TBX3 genes were cloned into the GLuc Dual-luciferase reporter vector and cotransfected with miR-17-92 or miR-Ctrl mimics $\left(n=3 ;{ }^{*} p<0.05\right)$. Luciferase activity was measured and normalised to Renilla luciferase activity $\left(n=4 ;{ }^{*} p<0.05\right)$. (F) Luciferase activity of $p C A G A 12-l u c$ SMAD4 reporter after stimulation with NODAL, ACTIVIN and TGF- $\beta 1$ in cells overexpressing miR-17-92 versus control cells. (G) RTgPCR analysis of p21 and TBX3 mRNA expression (upper panel) and western blot analysis for pSMAD2, p21 and GAPDH (lower panel) after stimulation with TGF- $\beta 1$, NODAL and ACTIVIN in cells overexpressing miR-17-92 versus control cells. 
To further show a direct interaction between miR-17-92 members and their targets, we assessed the ability of the cluster members to interact with the $3^{\prime}$ UTR of Alk4 and Tbx 3 using luciferase reporter constructs. The complete $3^{\prime}$ UTR of the Alk4 or Tbx3 gene were cloned into the GLuc Dual-luciferase reporter vector and CSCs were cotransfected with GLuc vectors containing the $3^{\prime}$ UTR of Alk4 or Tbx3 and miR-17-92 mimics. As expected, we found significantly lower luciferase expression in miR-17-92 CSCs (figure 5E). To functionally validate SMAD4 as a direct target of miR-17-92, we also used a pCAGA12-luciferase SMAD4 reporter. After stimulation with TGF- $\beta 1$, NODAL or ACTIVIN, we observed an increase in luciferase expression in miR-Ctrl cells, while in cells overexpressing miR-17-92 the effect was significantly diminished, indicating that SMAD4 is strongly inhibited by miR-17-92 (figure 5F) and suggesting that elevated miR-17-92 levels in bulk PDAC tumour cells could contribute to loss of SMAD4 expression in bulk PDAC samples. ${ }^{28}$ As it has been reported that TGF- $\beta 1$-mediated cancer cell migration and invasion is dependent on $\mathrm{p} 21,{ }^{29}$ we stimulated cells with TGF- $\beta 1$, NODAL or ACTIVIN to evaluate the induction of $\mathrm{p} 21, \mathrm{Tbx} 3$ and $\mathrm{pSMAD} 2$. In control cells, we observed increased expression of p21, TBX3 and pSMAD2, while in cells overexpressing miR-17-92, this effect was abrogated (figure 5G).

\section{Knockdown of p21 inhibits CSC phenotypes including chemoresistance}

The results presented above suggested that the targets of the miR-17-92 cluster are essential for CSCs. While we have shown previously that the miR-17-92 target Alk4 is crucial for selfrenewal of CSCs, we therefore focused on p21 as it was specifically downregulated in chemoresistant CSCs (figure 2A). Knock down of p21 using short-hairpin RNAs (sh-p21) (figure 6A) resulted in reduced sphere formation capacity during serial passaging (figure 6B), even though sh-p21 cells showed increased overall proliferation as determined by cell cycle analysis (figure 6C). As these in vitro data suggest an important role for p21 in CSCs biology, we next validated our findings in vivo. Limiting dilution assays using sh-scramble and sh-p21 CSCs revealed significantly reduced in vivo tumourigenicity, particularly when low numbers of cells were injected (figure 6D). Moreover, sh-p21 cells lost their invasive capacity (see online supplementary figure S6A) and became chemoresistant to gemcitabine (figure 6E). Thus, by merely silencing $\mathrm{p} 21$, one of the many targets of the miR-17-92 cluster, we were able to mimic, at least partially, the phenotypes of miR-17-92 CSCs.

\section{Knockdown of TBX3 inhibits CSC phenotypes}

Next, we studied the role of TBX3 in CSCs based on its essential role in embryogenesis and stem cell function ${ }^{30}$ as well as its implication in oncogenic processes. ${ }^{31-33}$ Indeed, TBX3 was consistently upregulated in sphere-derived CSCs at the mRNA (see online supplementary figure S6B) and protein levels (figure $6 \mathrm{~F}$, left panel). Consistently, TBX3 was significantly increased in CSCs defined as CD133+ (see online supplementary figure $\mathrm{S6C}$ ) or ALK4+ (see online supplementary figure S6D), and subsequent TBX3 knockdown (figure 6F, right panel) resulted in a significant reduction of CSC surface markers (figure 6G) and impaired sphere formation capacity (figure $6 \mathrm{H}$ ). Very recently, TBX3 was identified as a TGF- $\beta 1$ target with a dual function: inhibition of proliferation and promotion of the migration of breast epithelial cells. ${ }^{34}$ Indeed, silencing of TBX3 in CSCs also abrogated their invasive capacity, specifically when stimulated with NODAL, ACTIVIN or TGF- $\beta 1$ (see online supplementary figure S6E). Thus, like p21, TBX3 also represents a crucial factor through which the miR-17-92 cluster negatively regulates CSC phenotypes.

\section{Targeting quiescent CSCs as a novel therapeutic approach for PDAC}

Finally, we aimed to provide proof of concept for the translational relevance of our findings and performed in vivo therapeutic intervention studies by induced overexpression of miR-17-92 in established PDAC models using a doxycyclineswitchable system. Once tumours had formed $\left(\sim 100 \mathrm{~mm}^{3}\right)$, doxycycline was administered to induce miR-17-92 expression. Some mice also received gemcitabine (biweekly $125 \mathrm{mg} / \mathrm{kg}$ intraperitoneally) from day 14 to day 63 , to mimic standard of care. No significant differences were observed between miR-Ctrl and miR-17-92 tumours with respect to bulk tumour growth, which was expected based on the extensive proliferative capacity of bulk tumour cells. Intriguingly, however, miR-17-92 tumours were significantly more sensitive to gemcitabine (see figure 7A and online supplementary figure S7A) or Abraxane (see online supplementary figure S7B). Weight assessment (figure 7B) and flow cytometry analysis revealed a significant decrease in CSC content for miR-17-92 tumours treated with gemcitabine (figure 7C). Consistently, limiting dilution tumourigenicity assays showed reduced in vivo tumourigenicity for cells overexpressing miR-17-92 alone and additionally treated with gemcitabine, indicating that high levels of miR17-92 forced CSCs into a more differentiated and proliferative state, reducing their self-renewal capacity in vivo and at the same time sensitising them to gemcitabine (figure 7D).

\section{CONCLUSION/DISCUSSION}

Pancreatic cancers contain a rare population of undifferentiated 'stem-like' cells that are highly tumourigenic and give rise to more differentiated progenies. ${ }^{5} 6$ Mechanistically, these cells have activated pathways distinct from their non-differentiated counterparts, including activated NODAL/ACTIVIN signalling, which we have previously identified as an essential regulator of CSC 'stemness'. ${ }^{16}$ As CSCs and their more differentiated progenies share the same genetic background, we hypothesised that the apparent mechanistic differences that exist between CSCs and non-CSCs are related to a unique epigenetic signature. As such, we aimed to better characterise the epigenetic regulatory machinery of pancreatic CSCs using complementary approaches. Intriguingly, we were able to identify a specific and common miRNA signature present in treatment-naïve CSCs and gemcitabine-resistant CSCs isolated from a representative set of PDXs.

Specifically, we found the miR-17-92 cluster, composed of six members miR-17, miR-18a, miR-19a, miR-19b, miR-20a and miR-92a, to be markedly suppressed in pancreatic CSCs as compared with their more differentiated and chemosensitive counterparts. Interestingly, the miR-17-92 cluster has been validated as an oncogenic regulator and at the same time as a tumour suppressive regulator. This dual role can be explained, in part, by the spectrum and diversity of the mRNAs targeted by this cluster and the tumour and/or cellular context. While miR-17-92 has been shown to serve as an oncogene in some human cancers, ${ }^{35}{ }^{36}$ loss of heterozygosity at 13q12-q13 has also been linked to accelerated tumour progression and poor outcome in breast cancer, squamous cell carcinoma of the larynx, retinoblastoma, hepatocellular carcinoma and nasopharyngeal carcinoma, ${ }^{37} 38$ and deletion of the miR-17-92 cluster has been observed in a subset of patients with ovarian cancer, 
A

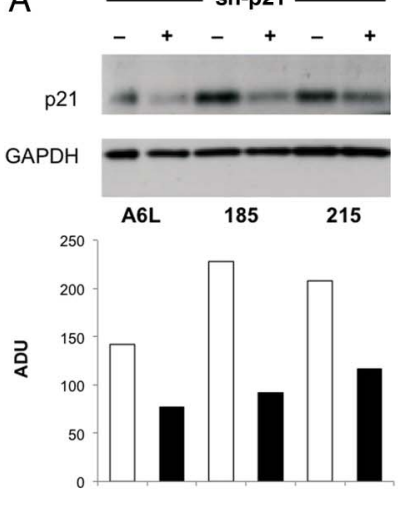

B

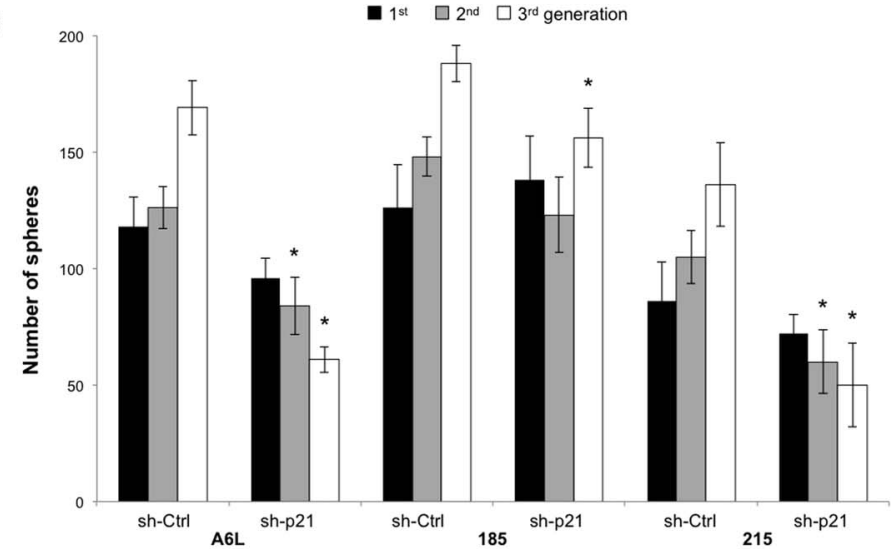

C

$$
\text { - } \mathrm{G}_{0} \square \mathrm{G}_{1} \square \mathrm{S} \quad \square \mathrm{G} 2 / \mathrm{M}
$$
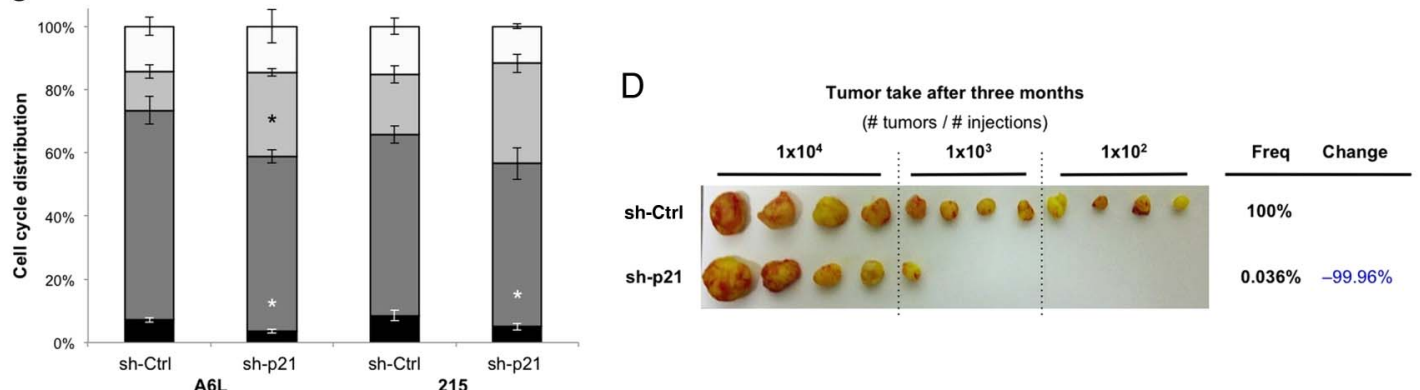

E

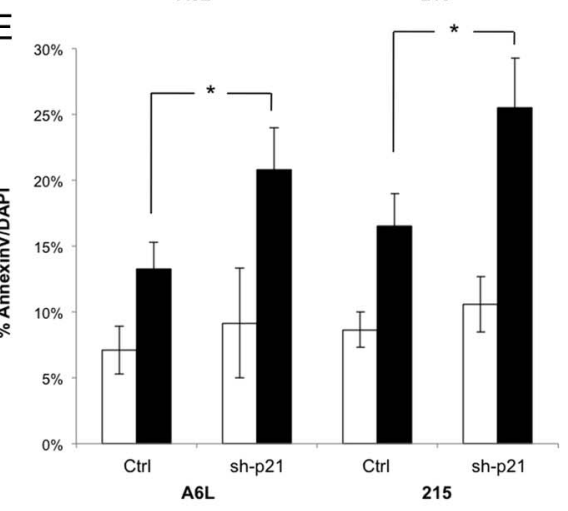

$\mathrm{F}$
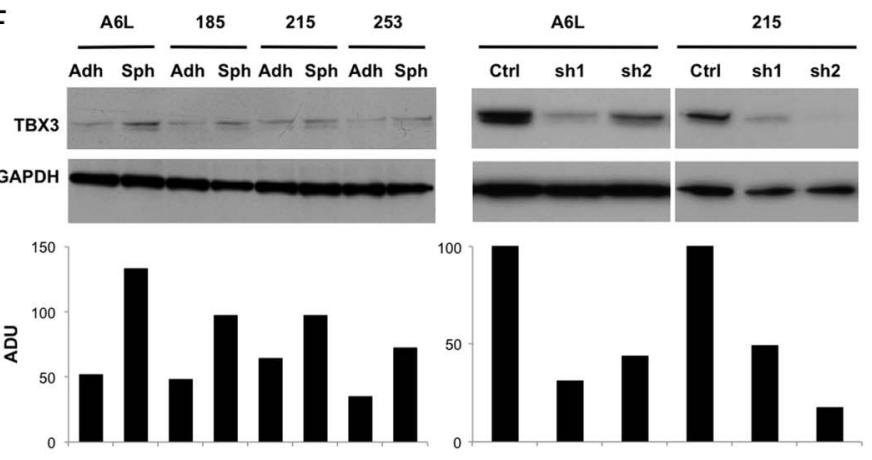

G
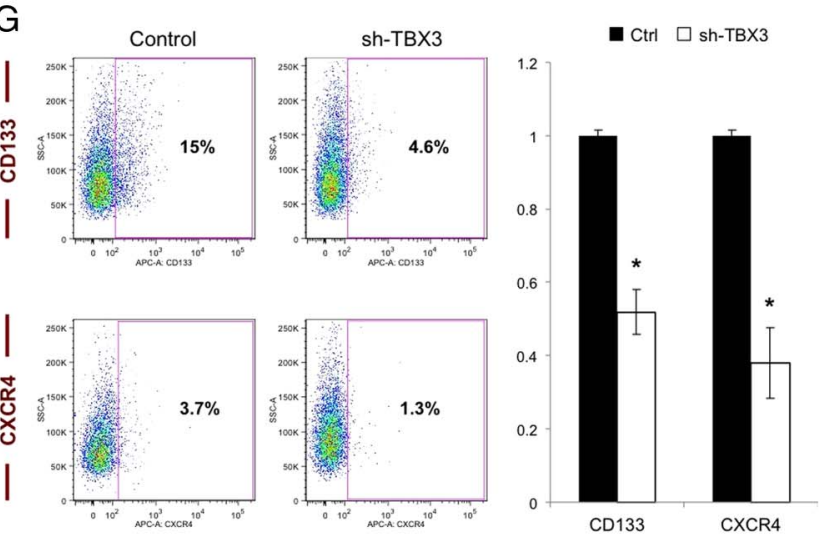

$\mathrm{H}$
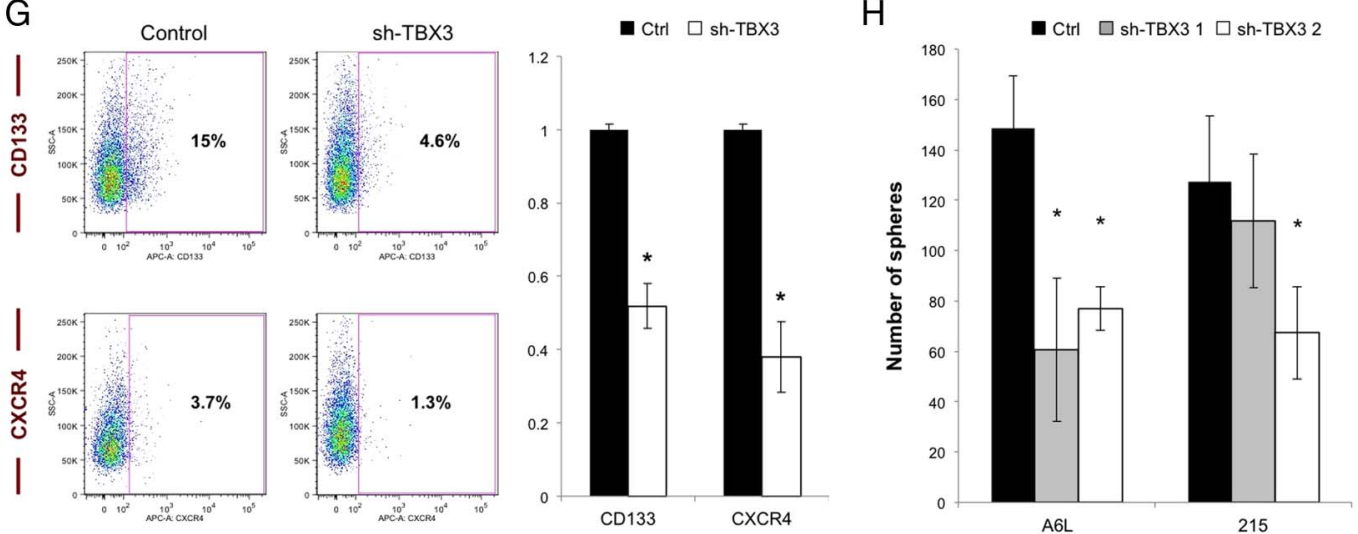

Figure 6 Knockdown of p21 or TBX3 inhibits cancer stem cell (CSC) phenotypes. (A-H) CSCs were stably infected with lentiviruses expressing a scrambled control shRNA (Ctrl), an shRNA against p21 (sh-p21) or an shRNA against TBX3 (sh-TBX3). (A) Western blot analysis of p21 in infected cells. ADU, arbitrary densitometric unit. (B) Serial sphere formation capacity of sh-p21-infected pancreatic ductal adenocarcinoma (PDAC) cells ( $n=3$; $\left.{ }^{*} p<0.05\right)$. (C) Cell cycle analysis using Ki67 staining $\left(n=3 ;{ }^{*} p<0.05\right)$. (D) Limiting dilution tumourigenicity analysis of sh-p21-infected 185 cells. Freq $=\operatorname{CSC}$ frequency $\left({ }^{*} p<0.05\right)$. (E) Chemoresistance to gemcitabine as determined with AnnexinVIDAPI staining after 7 days treatment $(n=3$; ${ }^{*} \mathrm{p}<0.05$ ). (F) Comparative western blot analysis of TBX3 protein levels in adherent (Adh) and sphere (Sph) from several primary PDAC cultures and densitometric quantification (left panel). Western blot analysis for TBX3 in cells expressing sh-Tbx3 (sh1 and sh2) and densitometric quantification (right panel). (G) Flow cytometry analysis for CSC surface markers CD133 and CXCR4 $\left(n=3 ;{ }^{*} p<0.05\right)$. (H) Sphere formation capacity of sh-TBX3-infected PDAC cells $\left(n=3 ;{ }^{*} p<0.05\right)$. 
A

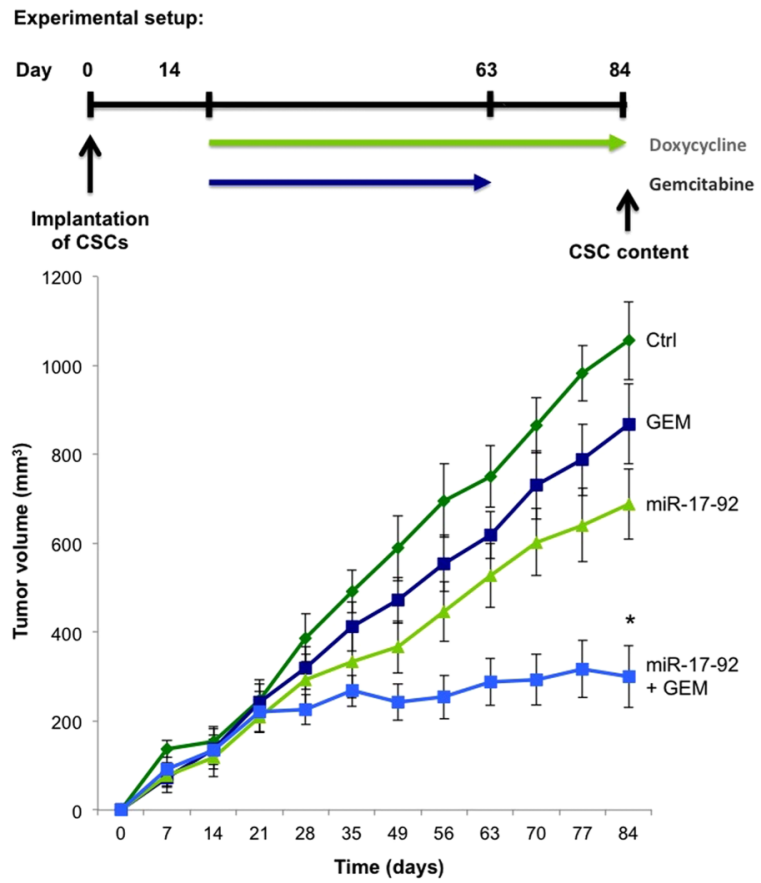

D
B
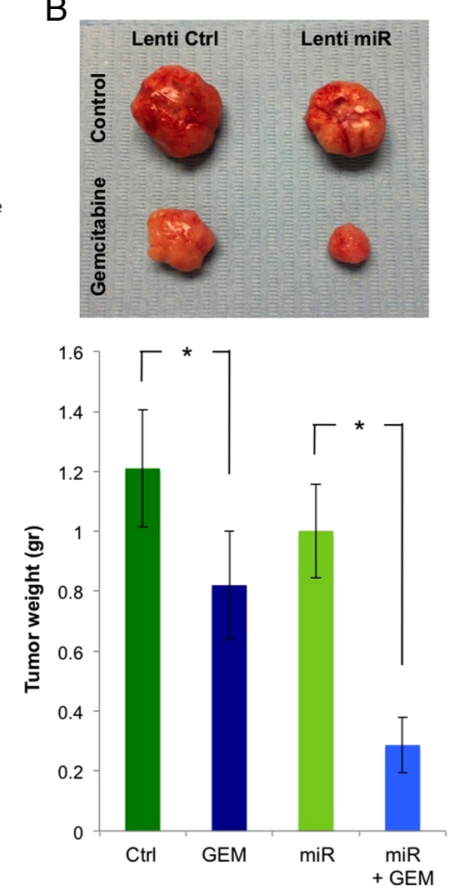

C
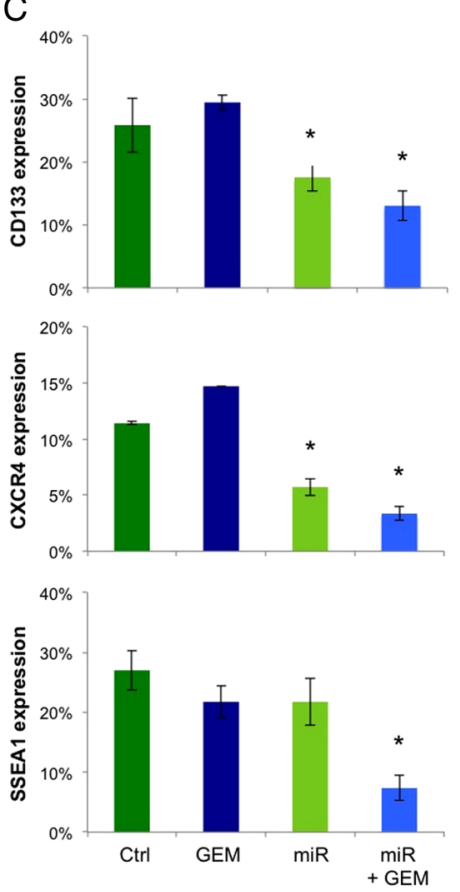

Figure 7 Targeting quiescent cancer stem cells (CSCs) as a novel therapeutic approach for pancreatic ductal adenocarcinoma (PDAC). (A) Experimental set-up for in vivo treatment (upper panel) and treatment effects of gemcitabine (GEM) in 185 patient-derived xenograft (PDX) tumours with or without induction of miR-17-92 expression (lower panel). The mean tumour volumes are reported ( $n=6$ tumours per group; $\left.{ }^{*} \mathrm{p}<0.05\right)$. (B) Representative picture (upper panel) and quantification of tumour weight (lower panel; $\left.n=6 ;{ }^{*} p<0.05\right)$.). (C) Flow cytometry analysis of CSC markers CD133, CXCR4 and SSEA1 in cells isolated from resected tumours on d $84\left(n=4 ;{ }^{*} p<0.05\right)$. (D) Limiting dilution tumourigenicity analysis of PDAC cells isolated from $d 84$ tumours $\left({ }^{*} p<0.05\right)$.

breast cancer and melanoma. ${ }^{39}$ Moreover, the role of the miR-17-92 cluster as a tumour suppressor has been demonstrated in breast cancer cells, ${ }^{40}$ in GI stromal tumours ${ }^{41}$ and in oral squamous carcinoma. ${ }^{42}$ Thus, the duality of this cluster reflects the complexities of cancer progression as well as the intricacies of the regulation network of miRNAs and their targets in a tumour and cell type-dependent manner.

Our data now demonstrate that targeted inhibition of miR-17-92 in pancreatic non-CSCs equipped these cells with features that are inherently restricted to bona fide CSCs, such as upregulation of CD133, increased sphere formation capacity, reduced proliferation and subsequent in vitro chemoresistance, and more importantly, increased in vivo tumourigenicity and in vivo resistance to chemotherapy. In contrast, overexpression of miR-17-92 in CSCs resulted in loss of stem-like features, including reduced self-renewal (ie, sphere formation capacity), and decreased expression of CSC surface markers, but also increased proliferation. The latter was most intriguing as it resulted in exhaustion of normally slow-cycling CSCs, which was reflected in decreased in vivo tumourigenicity during serial passaging and increased sensitivity to gemcitabine and Abraxane, respectively. Thus, our data demonstrate that suppression of miR-17-92 is crucial for maintaining the stemness phenotype in CSCs, while the apparent overexpression in non-CSCs equips these cells with extensive, albeit not indefinite, proliferative capacity.

Several members of the miR-17-92 cluster have been shown to target diverse pathways, including TGF- $\beta 1^{43}{ }^{44}$ and HIF-1 $\alpha$ signalling, ${ }^{45}$ but their role in the regulation of pancreatic cancer and specifically of pancreatic CSCs remained to be determined. Intriguingly, we were able to identify several targets clustering around pathways that have been previously associated with CSCs regulation and 'stemness' (eg, NODAL/ACTIVIN pathway and cell cycle regulators). We found miR-18a to regulate SMAD2 and SMAD4, two key components of the NODAL/ ACTIVIN signalling cascade. miR-17 and miR-20a targeted a wider range of genes including TGFBR2, ALK4, SMAD4, as well p21 and TBX3, all of which are again linked to NODAL/ ACTIVIN/TGF- $\beta$ signaling ${ }^{16}{ }^{44}$ and cell cycle regulation. ${ }^{24} 29$ Finally, miR-92a was found to also target TBX3 as well as p57. The sum of these data therefore suggests that miR-17-92 specifically dampens NODAL/ACTIVIN signalling in a multifaceted way by acting upstream and downstream of pSMAD2/SMAD4.

As the biological relevance of targeting NODAL/ACTIVIN and ALK4 in CSCs has already been demonstrated by our laboratory, ${ }^{16}$ herein we focused our efforts on the other less characteristic miR-17-92 targets p21 and TBX3. For p21, we 
observed that this cell cycle regulator was overexpressed in chemoresistant CSCs suggesting an important role for p21 in controlling CSC proliferation. Indeed, there is strong evidence from murine models of normal haematopoietic and leukaemic stem cells that p21 is an important regulator of self-renewal. In the absence of p21, normal haematopoietic stem cells as well as transformed leukaemic stem cells functionally exhaust over time. ${ }^{25}{ }^{46}$ Our data now also support a role for p21 in the prevention of pancreatic CSC exhaustion through cell cycle restriction. Inhibiting p21 in CSCs resulted in impaired self-renewal and chemoresistance. the accumulation of DNA damage as evidenced by increased gH2AX foci and their subsequent elimination/dysfunction likely via mitotic chaos.

TBX3 is a transcription factor that belongs to the T-box gene family and contains a conserved DNA-binding domain called the T-box. ${ }^{47}$ TBX3 plays an important role in embryonic development, cell cycle regulation and cancer progression. ${ }^{27}{ }^{33}$ For example, overexpressing TBX3 in non-tumourigenic early stage melanoma cells promoted tumour formation and invasion. Furthermore, it has been shown that TBX3 functions as a reciprocal switch between substrate-dependent cell proliferation and tumour invasion. ${ }^{33}$ TBX3 also plays a pivotal role in TGF- $\beta 1$-mediated antiproliferation and promigration ${ }^{34}$ and in the regulation of NODAL signalling in embryonic stem cells. ${ }^{48}$ Here we now show that TBX3 is upregulated in CSCs and its knockdown negatively affects CSC phenotypes via TGF- $\beta 1$ dependent and NODAL-dependent mechanisms. Thus, our data demonstrate the importance of TBX3 in the regulation of pancreatic CSCs self-renewal and metastasis via regulation of TGF- $\beta 1$ and NODAL signalling.

In summary, our study has identified the miR-17-92 cluster as a previously unknown negative master regulator of pancreatic CSCs. While we are still investigating why this cluster is differentially expressed in CSCs versus non-CSCs, we have observed that MYC, a known regulator of the miR-17-92 cluster, ${ }^{36} 3843$ is consistently downregulated in pancreatic CSCs. More importantly, we demonstrate, for the first time, that the miR-17-92 cluster regulates CSCs via targeting ALK4, p21 and TBX3. Overexpression of miR-17-92 led to abrogation of CSC phenotypes and eventual loss of in vivo tumourigenicity, whereas suppression of miR-17-92 contributed to PDAC aggressiveness including invasiveness. In addition, it is important to note that the simultaneous targeting of the different components of the NODAL/ACTIVIN signalling cascade as well as its downstream effectors through multiple miRNAs of the miR-17-92 cluster allows for a very tight control of this transcriptional programme. Thus, from a clinical point of view, targeting pancreatic CSCs using the multifaceted effects of miR-17-92 on NODAL/ ACTIVIN signalling could be a promising and highly specific therapeutic approach, as it would result in reduced expression of ALK4, and induce direct miR-17-92-mediated repression of NODAL/ACTIVIN responsive genes. The latter would avoid the bias of the SMAD4 status as Smad4 is mutated in about 50\% of pancreatic tumours, ${ }^{49}$ even though these tumours still maintain active NODAL/ACTIVIN signalling as a key regulator of their self-renewal capacity. ${ }^{16}$ Indeed, the plasmid-based production of modified miRNA, which also allows for the generation of polycistronic constructs such as miR-17-92, ${ }^{50}$ could represent an important next step to further evaluate this novel therapeutic concept in preclinical models of PDAC.

Acknowledgements The authors thank Sonia Alcala for excellent technical assistance and Dr Aristidis Moustakas (Ludwig Cancer Research Centre, Uppsala, Sweden) for providing the pCAGA12-luc SMAD4 reporter.
Contributors MC developed the study concept, acquired, analysed and interpreted data as well as drafted the manuscript; YS-R, SMT, IM-L, EL, JD and AA acquired and analysed data; CRV and JCR designed and developed all viruses used in the study; SH performed and analysed the miRNA microarray experiments; MH provided extensively characterised PDAC samples; BSJr developed the study concept, interpreted the data and wrote the manuscript; $\mathrm{CH}$ developed the study concept, obtained funding, interpreted the data and wrote the manuscript.

Funding CH: ERC Advanced Investigator Grant (Pa-CSC 233460), European Community's Seventh Framework Programme (FP7/2007-2013) under grant agreement No 256974 (EPC-TM-NET) and No 602783 (CAM-PaC), the Subdirección General de Evaluación y Fomento de la Investigación, Fondo de Investigación Sanitaria (PS09/02129 \& PI12/02643), and the Programa Nacional de Internacionalización de la I+D, Subprogramma: FCCI 2009 (PLE2009-0105; Ministerio de Economía y Competitividad, Spain). MC: La Caixa Predoctoral Fellowship.

Competing interests None.

Patient consent Obtained.

Ethics approval Instituto de Salud Carlos III, Madrid, Spain.

Provenance and peer review Not commissioned; externally peer reviewed.

Data sharing statement No additional unpublished data from the study are available.

Open Access This is an Open Access article distributed in accordance with the Creative Commons Attribution Non Commercial (CC BY-NC 4.0) license, which permits others to distribute, remix, adapt, build upon this work non-commercially, and license their derivative works on different terms, provided the original work is properly cited and the use is non-commercial. See: http://creativecommons.org/ licenses/by-nc/4.0/

\section{REFERENCES}

1 Siegel R, Naishadham D, Jemal A. Cancer statistics, 2012. CA Cancer J Clin 2012;62:10-29.

2 Rahib L, Smith BD, Aizenberg R, et al. Projecting cancer incidence and deaths to 2030: the unexpected burden of thyroid, liver, and pancreas cancers in the United States. Cancer Res 2014;74:2913-21.

3 Von Hoff DD, Ervin T, Arena FP, et al. Increased survival in pancreatic cancer with nab-paclitaxel plus gemcitabine. N Engl J Med 2013;369:1691-703.

4 Visvader JE, Lindeman GJ. Cancer stem cells in solid tumours: accumulating evidence and unresolved questions. Nat Rev Cancer 2008:8:755-68.

5 Hermann PC, Huber SL, Herrler T, et al. Distinct populations of cancer stem cells determine tumor growth and metastatic activity in human pancreatic cancer. Cell Stem Cell 2007;1:313-23.

6 Li C, Heidt DG, Dalerba $P$, et al. Identification of pancreatic cancer stem cells. Cancer Res 2007:67:1030-7.

7 Croce CM, Calin GA. miRNAs, cancer, and stem cell division. Cell 2005;122:6-7.

8 Melton C, Judson RL, Blelloch R. Opposing microRNA families regulate self-renewal in mouse embryonic stem cells. Nature 2010;463:621-6.

9 Yu F, Yao H, Zhu $\mathrm{P}$, et al. let-7 regulates self renewal and tumourigenicity of breast cancer cells. Cell 2007;131:1109-23.

10 Shimono Y, Zabala M, Cho RW, et al. Downregulation of miRNA-200c links breast cancer stem cells with normal stem cells. Cell 2009;138:592-603.

11 Hidalgo $\mathrm{M}$, Amant $\mathrm{F}$, Biankin AV, et al. Patient-derived xenograft models: an emerging platform for translational cancer research. Cancer Discov 2014:4:998-1013.

12 Jimeno A, Feldmann G, Suarez-Gauthier A, et al. A direct pancreatic cancer xenograft model as a platform for cancer stem cell therapeutic development. Mol Cancer Ther 2009:8:310-14.

13 Jones $S$, Zhang $X$, Parsons DW, et al. Core signaling pathways in human pancreatic cancers revealed by global genomic analyses. Science 2008;321:1801-6.

14 Mueller MT, Hermann PC, Witthauer J, et al. Combined targeted treatment to eliminate tumourigenic cancer stem cells in human pancreatic cancer. Gastroenterology 2009;137:1102-13.

15 Balic A, Dræby-Sørensen M, Trabulo SM, et al. Chloroquine targets pancreatic cancer stem cells via inhibition of CXCR4 and hedgehog signaling. Mol Cancer Ther 2014;13:1758-71.

16 Lonardo E, Hermann PC, Mueller MT, et al. Nodal/Activin signaling drives self-renewal and tumourigenicity of pancreatic cancer stem cells and provides a target for combined drug therapy. Cell Stem Cell 2011;9:433-46.

17 de Wolf $C$, Jansen $\mathrm{R}$, Yamaguchi $\mathrm{H}$, et al. Contribution of the drug transporter ABCG2 (breast cancer resistance protein) to resistance against anticancer nucleosides. Mol Cancer Ther 2008;7:3092-102.

18 Sharom FJ. $A B C$ multidrug transporters: structure, function and role in chemoresistance. Pharmacogenomics 2008;9:105-27.

19 Santini D, Vincenzi B, Fratto ME, et al. Prognostic role of human equilibrative transporter 1 (hENT1) in patients with resected gastric cancer. J Cell Physiol 2010;223:384-8. 
20 Pece $\mathrm{S}$, Tosoni $\mathrm{D}$, Confalonieri $\mathrm{S}$, et al. Biological and molecular heterogeneity of breast cancers correlates with their cancer stem cell content. Cell 2010;140:62-73.

21 Roesch A, Fukunaga-Kalabis M, Schmidt EC, et al. A temporarily distinct subpopulation of slow-cycling melanoma cells is required for continuous tumor growth. Cell 2010;141:583-94.

22 Volinia S, Calin GA, Liu CG, et al. A microRNA expression signature of human solid tumors defines cancer gene targets. Proc Natl Acad Sci USA 2006;103:2257-61.

23 Matsumoto A, Takeishi S, Kanie T, et al. p57 is required for quiescence and maintenance of adult hematopoietic stem cells. Cell Stem Cell 2011;9:262-71.

24 Kippin TE, Martens DJ, van der Kooy D. p21 loss compromises the relative quiescence of forebrain stem cell proliferation leading to exhaustion of their proliferation capacity. Genes Dev 2005;19:756-67.

25 Cheng $T$, Rodrigues $N$, Shen $\mathrm{H}$, et al. Hematopoietic stem cell quiescence maintained by p21cip1/waf1. Science 2000;287:1804-8.

26 Lu R, Yang A, Jin Y. Dual functions of T-box 3 (Tbx3) in the control of self-renewal and extraembryonic endoderm differentiation in mouse embryonic stem cells. J Biol Chem 2011;286:8425-36.

27 Fillmore CM, Gupta PB, Rudnick JA, et al. Estrogen expands breast cancer stem-like cells through paracrine FGF/Tbx3 signaling. Proc Natl Acad Sci USA 2010;107:21737-42.

28 Wilentz RE, lacobuzio-Donahue CA, Argani P, et al. Loss of expression of Dpc4 in pancreatic intraepithelial neoplasia: evidence that DPC4 inactivation occurs late in neoplastic progression. Cancer Res 2000;60:2002-6.

29 Dai M, Al-Odaini AA, Arakelian A, et al. A novel function for p21Cip1 and acetyltransferase $\mathrm{p} / \mathrm{CAF}$ as critical transcriptional regulators of TGFbeta-mediated breast cancer cell migration and invasion. Breast Cancer Res 2012;14:R127.

30 Pirity MK, Dinnyes A. Tbx3: another important piece fitted into the pluripotent stem cell puzzle. Stem Cell Res Ther 2010;1:12.

31 Renard CA, Labalette C, Armengol C, et al. Tbx3 is a downstream target of the Wnt/beta-catenin pathway and a critical mediator of beta-catenin survival functions in liver cancer. Cancer Res 2007;67:901-10.

32 Fan $X$, Matsui W, Khaki L, et al. Notch pathway inhibition depletes stem-like cells and blocks engraftment in embryonal brain tumors. Cancer Res 2006;66:7445-52.

33 Peres J, Prince $S$. The T-box transcription factor, TBX3, is sufficient to promote melanoma formation and invasion. Mol Cancer 2013;12:117.

34 Li J, Weinberg MS, Zerbini L, et al. The oncogenic TBX3 is a downstream target and mediator of the TGF-beta1 signaling pathway. Mol Biol Cell 2013;24:3569-76.

35 He L, Thomson JM, Hemann MT, et al. A microRNA polycistron as a potential human oncogene. Nature 2005;435:828-33.
36 O'Donnell KA, Wentzel EA, Zeller KI, et al. c-Myc-regulated microRNAs modulate E2F1 expression. Nature 2005;435:839-43.

37 Xiang J, Wu J. Feud or Friend? The Role of the miR-17-92 Cluster in Tumorigenesis Curr Genomics 2010;11:129-35.

38 Coller HA, Forman JJ, Legesse-Miller A. "Myc'ed messages": myc induces transcription of E2F1 while inhibiting its translation via a microRNA polycistron. PLoS Genet 2007;3:e146.

39 Zhang $\mathrm{L}$, Huang J, Yang $\mathrm{N}$, et al. microRNAs exhibit high frequency genomic alterations in human cancer. Proc Natl Acad Sci USA 2006;103: 9136-41.

40 Hossain A, Kuo MT, Saunders GF. Mir-17-5p regulates breast cancer cell proliferation by inhibiting translation of AIB1 mRNA. Mol Cell Biol 2006;26:8191-201.

41 Gits CM, van Kuijk PF, Jonkers MB, et al. MiR-17-92 and miR-221/222 cluster members target KIT and ETV1 in human gastrointestinal stromal tumours. $\mathrm{Br} J$ Cancer 2013;109:1625-35.

42 Chang CC, Yang YJ, Li YJ, et al. MicroRNA-17/20a functions to inhibit cell migration and can be used a prognostic marker in oral squamous cell carcinoma. Oral Oncol 2013;49:923-31.

43 Dews M, Fox JL, Hultine S, et al. The myc-miR-17 92 axis blunts TGF\{beta\} signaling and production of multiple TGF\{beta\}-dependent antiangiogenic factors. Cancer Res 2010;70:8233-46.

44 Mestdagh P, Bostrom AK, Impens F, et al. The miR-17-92 microRNA cluster regulates multiple components of the TGF-beta pathway in neuroblastoma. $\mathrm{Mol}$ Cell 2010;40:762-73.

45 Taguchi A, Yanagisawa K, Tanaka M, et al. Identification of hypoxia-inducible factor-1 alpha as a novel target for miR-17-92 microRNA cluster. Cancer Res 2008:68:5540-5.

46 Viale A, De Franco F, Orleth A, et al. Cell-cycle restriction limits DNA damage and maintains self-renewal of leukaemia stem cells. Nature 2009;457:51-6.

47 Bamshad M, Lin RC, Law DJ, et al. Mutations in human TBX3 alter limb, apocrine and genital development in ulnar-mammary syndrome. Nat Genet 1997;16: 311-15.

48 Weidgang CE, Russell R, Tata PR, et al. TBX3 Directs Cell-Fate Decision toward Mesendoderm. Stem Cell Reports 2013;1:248-65.

49 Schneider G, Schmid RM. Genetic alterations in pancreatic carcinoma. Mol Cancer 2003;2:15.

50 Lee J, Sayed $N$, Hunter $A$, et al. Activation of innate immunity is required for efficient nuclear reprogramming. Cell 2012;151:547-58. 


\section{Erratum: The miR-17-92 cluster counteracts quiescence and chemoresistance in a distinct subpopulation of pancreatic cancer stem cells}

Cioffi M, Trabulo SM, Sanchez-Ripoll Y, et al. The miR-17-92 cluster counteracts quiescence and chemoresistance in a distinct subpopulation of pancreatic cancer stem cells. Gut 2015;64:1936-48. Two errors have been corrected in the text. In the paragraph "Knockdown of p21 inhibits CSC phenotypes including chemoresistance", line 5 has been corrected to “... we therefore focused on p21 as it was specifically upregulated in chemoresistant CSCs (figure $2 \mathrm{~A})$ ". In the same paragraph, line 16 has been corrected to "and became chemosensitive to gemcitabine (figure 6E)".

Gut 2016;65:1224. doi:10.1136/gutjnl-2014-308470corr1 